\title{
Induced-charge electrokinetic (ICEK) development and applications in microfluidics
}

\author{
Mohammad Karim Dehghan Manshadi ${ }^{1}$, Mehdi Mohammadi ${ }^{1}$, Mohammad Zarei ${ }^{2}$, mahsa \\ saadat $^{3}$, and Amir Sanati Nezhad ${ }^{4}$ \\ ${ }^{1}$ University of Calgary Schulich School of Engineering \\ ${ }^{2}$ University of Pennsylvania \\ ${ }^{3}$ Kerman University of Medical Sciences \\ ${ }^{4}$ University of Calgary
}

May 5, 2020

\begin{abstract}
Applying an external electric field over a polarizable electrode or object within microchannels can induce an electric double layer (EDL) around channel walls and create induced-charge electrokinetics (ICEK) within channels. The primary consequence of the induced charge is the generation of micro-vortices around the polarizable electrode or object, presenting a great potential for various microfluidic applications. This review presents the advances in theoretical, numerical and experimental studies on the physics and applications of ICEK within microfluidics. In particular, the characteristics and performance of ICEK-based microfluidic components in active micromixers, micropumps, and microvalves are critically reviewed, followed by discussing the applications of ICEK in electrophoresis and particle/cell manipulation within microfluidics. Furthermore, the opportunities and challenges of ICEK-based microfluidic devices are highlighted. This work facilitates in recognizing deliverable ICEK-based microfluidic technologies with unprecedented functionality for the next generation of biomedical applications with predictable manufacturability and functionality.
\end{abstract}

\section{Introduction}

Lab-on-chip (LOC) microfluidic devices have been developed for various biomedical and biochemical applications (Azarmanesh et al., 2019; Sackmann, Fulton, \& Beebe, 2014; Shamsi, Mohammadi, Manshadi, \& Sanati-Nezhad, 2019; Jing Wu, He, Chen, \& Lin, 2016). Components, such as micromixers, micropumps, and microvalves are integrated into LOC devices to automate their function (Bhagat et al., 2010). While passive platforms operate without any external energy source, active systems operate mainly based on one of thermophoretic (Vigolo, Rusconi, Stone, \& Piazza, 2010), acoustophoretic (Barani et al., 2016; Lenshof, Magnusson, \& Laurell, 2012), magnetophoretic (S. Kim et al., 2013; M. K. Manshadi et al., 2018), electrokinetic (Mohammadi, Madadi, Casals-Terré, \& Sellarès, 2015; C. Zhao \& Yang, 2013) or centrifugal forces (Kinahan et al., 2016; Mohammadi, Kinahan, \& Ducrée, 2016; Tang, Wang, Kong, \& Ho, 2016). Electrokinetic techniques have become one of the most popular active methods for chemical analysis and biomedical diagnostics due to their inherent characteristics, including their high controllability, flat velocity profile in microchannels, independency form channel size, and function without need to moving parts (Cunlu Zhao \& Yang, 2012).

Electrokinetics refers to fluid or particle motion in liquid electrolytes under an external electric field (Bazant \& Squires, 2010). In classical electrokinetics, the main assumption is a linear response to the applied electric potential within microchannels that have constant surface charge (Todd M Squires \& Bazant, 2004). Therefore, classical electrokinetic phenomena are also called linear electrokinetics. However, the possible drawbacks 
of linear electrokinetics are: i) weak flow rate, ii) zero time-averaged liquid flow under alternating current (AC) electric fields, and iii) the need to a high electric potential along the microchannel (with the length order of centimeter) to generate a strong electric field strength (Bazant \& Squires, 2004, 2010). These drawbacks have been improved by introducing non-linear electrokinetics (Ajdari, 2000; Ramos, Morgan, Green, \& Castellanos, 1999). Such non-linearity was first examined by Ramos et al. (Ramos et al., 1999) around locally asymmetric polarizable electrodes where they observed AC electroosmotic (ACEO) fluid flow over a pair of adjacent metal microelectrodes fabricated on planar glass substrates (Ramos et al., 1999). Ajdari (Ajdari, 2000) further employed locally asymmetric arrays of electrodes to demonstrate low-voltage ACEO micro-pumping. Non-linear AC electrokinetics was further used for particle manipulation (Mirzajani et al., 2016), DNA concentration (Bown \& Meinhart, 2006), and micromixing (Sasaki, Kitamori, \& Kim, 2010).

Bazant and Squires (Bazant \& Squires, 2004) first generalized ACEO fluid flow to dielectric and conducting structures by introducing the term of induced-charge electroosmosis (ICEO) in a weak DC or AC electric field. The ICEO describes the interactions between the applied electric field and the induced ionic charge near the polarizable surface (electrode or object) (Todd M Squires \& Bazant, 2004). The induced-charge electrokinetic (ICEK) theory and applications, as well as theoretical and experimental advances on ICEK, were further presented (Bazant, Kilic, Storey, \& Ajdari, 2009; Bazant \& Squires, 2010). Then, Bazant (Bazant, 2011), in his book chapter, introduced the basic physical concepts of ICEK. Zhao and Yang's review article (Cunlu Zhao \& Yang, 2012) then focused on the breakthrough in electrokinetics and its physical mechanisms in micro and nanofluidics. Ramos et al. (Ramos, García-Sánchez, \& Morgan, 2016) studied AC electrokinetic characteristics of polarizable microparticles with a focus on the theory and experimental observations. The field of non-linear ICEK has expanded rapidly over the past few years to various microfluidic and LOC applications, such as induced-charge electrophoresis (ICEP) (Todd M Squires \& Bazant, 2006), ICEO micropump (Paustian, Pascall, Wilson, \& Squires, 2014), ICEO microvalve (C. Wang, Song, Pan, \& Li, 2016), ICEK micromixer (M. K. D. Manshadi, Khojasteh, Mansoorifar, \& Kamali, 2016; M. K. D. Manshadi, Nikookar, Saadat, \& Kamali, 2019), and recently in two-phase flow research studies (Bazant, 2015; W. Liu et al., 2017; Ren, Liu, Liu, et al., 2018).

This work reviews theoretical, numerical and experimental studies on the physics and applications of ICEK within microfluidics. The characteristics and performance of ICEK-based microfluidic components in active micromixers, micropumps, and microvalves are then reviewed, Furthermore, the opportunities and challenges of ICEK-based microfluidic devices are highlighted.

\section{Theory of ICEK within microfluidics}

In classic electrokinetics, the interaction between the applied electric field and the non-conducting microchannel walls results in a linear relationship between the induced zeta potential and the applied electric field (Z. Wu \& Li, 2008a). However, in non-linear ICEK, such correlation behaves differently. Theoretically, upon applying an electric field (E) around a polarizable object immersed in an electrolyte, the field lines intersect with the conducting surfaces (Todd M Squires \& Bazant, 2004). Due to the electrolyte's conductivity $(\sigma)$, a current of $\mathrm{J}=\sigma E$ is induced in the solution which therefore drives positive ions toward one side of the object and negative ions into the other side (Fig. 1A, i ). The ions movement continues until the field lines are expelled by the screening charge cloud around the object and the steady state condtion is achieved (Fig. $1 \mathrm{~A}$, ii ).

The changes in surface charge density $(q)$ over time for an ideal polarizable object with no electrochemical reactions at the solid/liquid interface is obtained from equation (1) (Todd M Squires \& Bazant, 2004).

$$
\overline{\frac{d q(\theta)}{\mathrm{dt}}=\sigma \mathbf{E} \bullet \mathbf{r} \quad(1)}
$$

where surface charge density $(q)$ and zeta-potential $(\zeta)$ have a linear relationship as equation (2).

$$
\overline{\zeta \equiv \frac{q}{e_{w} k} \quad(2)}
$$


where ew is dielectric permittivity of the solvent and $k-1(\lambda \Delta)$ is Debye screening length. Therefore, the change in zeta-potential over time until achieving a steady-state condition is obtained from equation (3).

$$
\overline{\frac{d \zeta(\theta)}{\mathrm{dt}}=\frac{\sigma}{e_{w} k} \mathbf{E} \bullet \mathbf{r} \quad(3)}
$$

In the steady-state mode, since the normal component of the electric field to the charge cloud is zero, its tangential component induces the ICEO flow. The ICEO around a polarizable object (Fig. 1A, iii ) or electrode (Fig. 1B ) can induce micro-vortices (MVs) around the object.

Helmholtz-Smoluchowski formula can be used to find the local slip ICEO velocity around the conducting object (equations 4) (Todd M Squires \& Bazant, 2004).

$$
\begin{aligned}
& \zeta_{i}=-\varnothing_{e}+\varnothing_{c} \\
& \varnothing_{c}=\frac{\int_{S} \varnothing_{e} \mathrm{dA}}{A}
\end{aligned}
$$

where $\mathbf{E t}$ is a tangential electric field, $\mu$ eo is electroosmotic mobility, and $\mu$ is fluid viscosity. Various equations have been proposed to define the induced charges over polarizable objects. For instance, equation (5) was derived to describe the correlation between the potential in the polarizable dielectric domain and the bulk of the fluid domain (Cunlu Zhao, 2012).

$$
\overline{\left.\lambda_{D} \frac{e_{d}}{e_{f}} n \bullet \nabla \Phi_{d}\right|_{n=0}=2 \frac{K_{B} T}{\mathrm{ze}} \sinh \left[\frac{z e\left(\left.\Phi_{f}\right|_{n=\lambda_{D}}-\left.\Phi_{d}\right|_{n=0}\right)}{2 K_{B} T}\right]+\zeta_{d 0} \quad(5)}
$$

where ed is dielectric constant of the dielectric block, ef is dielectric constant of the electrolyte, $\Phi \mathrm{d}$, and $\Phi \mathrm{f}$ are potentials in the bulk fluid and dielectric block, respectively, KB is Boltzmann constant, $\mathrm{T}$ is absolute temperature, e is elementary charge, $\mathrm{z}$ is valence of ions, and $\zeta \mathrm{d} 0$ is zeta potential corresponding to surface charge density (qd0). Wu and $\mathrm{Li}(\mathrm{Z}$. Wu \& Li, 2008b) suggested a numerical calculation for the induced zeta potential on the surface of an arbitrary polarizable object (equations 6).

where $\varnothing_{\mathrm{e}}$ is external electric potential and $\varnothing_{\mathrm{c}}$ is constant correction potential for a conducting surface with an area of A. This correction equation is based on the assumption that the conducting object is initially uncharged and the magnitude of the local strength of the induced field on the surface is equal to the externally applied electrical field magnitude with opposite directions. These correlations have been widely used to explain the ICEK phenomena employed in various microfluidics applications. In the following sections, these applications are discussed.

\section{Application of ICEK microfluidics}

ICEK-based microfluidic systems have contributed tremendously to developing different LOC components, such as micropumps, micromixers, microvalves, as well as particle manipulation and two-phase flow studies, which are addressed in this section.

\subsection{ICEK micropumps}

Inducing of controlled fluid flow in microchannels without the need for expensive and bulky injection systems is one of the hurdles of designing LOCs, particularly for developing miniaturized and high-throughput (HT) platforms (Dehghan Manshadi, Khojasteh, Mohammadi, \& Kamali, 2016). Electrokinetic-based micropumps 
have a high potential in maintaining the pressure drop along the microchannels due to their simple structure with no need for a moving part, easy fabrication, and integrability on a chip. More importantly, these pumps can generate continuous and precise flow with high controllability and long-term perfusion (Kamali, Manshadi, \& Mansoorifar, 2016; Zhou, Zhang, Li, \& Wang, 2016). Unlike the conventional electroosmotic flow (EOF) micropumps, ICEK micropumps induce fluid flow in microchannels deliberately with the capability of rapid change of fluid flow with minimal intertia by designing proper polarizable objects/electrodes in both DC and AC electric fields (Todd M. Squires, 2009). Here, the advances in ICEK micropumps are discussed, wherein these pumps are classified as ACEO-based (around polarizable electrodes) and ICEO-based (around polarizable objects) micropumps.

\subsubsection{ACEO micropumps}

ACEO phenomena employed non-linear electrokinetics by using a locally asymmetric polarizable electrode to induce fluid flow in microchannels (Ajdari, 2000). Brown et al. (Brown, Smith, \& Rennie, 2000) utilized a pair of planar electrodes with different edge sizes to induce fluid flow in channels. This work was further optimized by Studer et al. (V Studer, Pepin, Chen, \& Ajdari, 2002) to improve the flow control within microchannels (Fig. 2A ). This design configuration showed that breaking the electrode symmetry created an asymmetry charge distribution along the channel's axis, which led to a frequency-dependent pumping. The asymmetry pair of a planar electrode array was also used for micro-pumping thanks to its ease of fabrication, lowvoltage operation, and capability in local flow control (Jie Wu, 2008). Theoretical modeling of the frequencydependent pumping was further developed (Ramos, Gonzalez, Castellanos, Green, \& Morgan, 2003) and used to simulate different aspects of the ACEO pumping (Debesset, Hayden, Dalton, Eijkel, \& Manz, 2004; Vincent Studer, Pépin, Chen, \& Ajdari, 2004). Furthermore, the effect of several parameters and pumping configurations were investigated, such as studying the effect of channel height, electrochemical reactions, and non-linear surface capacitance of the Debye layer (Olesen, Bruus, \& Ajdari, 2006); controlling the pumping direction by switching the voltage using an inclined electrode array (Hilber, Weiss, Saeed, Holly, \& Jakoby, 2009; Loucaides, Ramos, \& Georghiou, 2007), pumping of two different electrolytes simultaneously through microchannels (Morgan, Green, Ramos, \& García-Sánchez, 2007), bubble-free pumping (Kuo \& Liu, 2008; Tawfik \& Diez, 2017) as well as ACEO pumping using biased AC/DC signals (Islam \& Reyna, 2012; Lian \& Wu, 2009; Piñón et al., 2017; Jie Wu, 2008; Yang Ng, Ramos, Cheong Lam, \& Rodriguez, 2012), pulse voltage waveforms (Tawfik \& Diez, 2017), square pole-slit electrode arrays (Yoshida, Sato, Eom, Kim, \& Yokota, 2017), and arrays of asymmetric ring electrode pairs in the cylindrical microchannels (Gao \& Li, 2018). Also, the comparison between fluid velocity on arrays of identical electrodes with $\mathrm{AC}$ voltage and a traveling-wave potential demonstrated that traveling-wave potential resulted in a higher fluid velocity (Ramos et al., 2005; Yang, Jiang, Ramos, \& García-Sánchez, 2009).

Besides using a planar array of electrodes, Urbanski et al. (Urbanski, Thorsen, Levitan, \& Bazant, 2006) introduced the first nonplanar ACEO pump by using three-dimensional (3D) stepped electrodes. They demonstrated the capability of higher flow rate pumping compared to planar ACEO pumps (Fig. 2B ). Senousy and Harnett (Senousy \& Harnett, 2010) introduced a less expensive and an easy fabrication method for constructing 3D stepped electrodes. Rouabah et al. (Rouabah et al., 2011) further studied the performance of a new set of 3D high-aspect-ratio electrodes for ACEO pumping while Huang et al. (C.-C. Huang, Bazant, \& Thorsen, 2010) employed these 3D electrode micropumps for liquid manipulation in portable biomedical microfluidic devices.

\subsubsection{ICEO micropumps}

Bazant and Squires (Bazant \& Squires, 2004; Todd M Squires \& Bazant, 2004) suggested that the ICEO flow around polarizable obstacles has the potential for liquid pumping in microchannels. Zhang et al. (Kai Zhang, Tian, \& Yu, 2012) numerically demonstrated that ICEO around conducting/Janus cylinders immersed in a microchannel could be used for efficient liquid pumping. Zhang et al. (Kai Zhang, Mi, \& Sheng, 2013) showed, in a numerical study, the capability of inducing the ICEO-based pumping by employing a Janus cylinder in a T-shape microchannel. Nobari et al. (Nobari, Movahed, Nourian, \& Kazemi, 2016) used a similar numerical strategy but using a conducting cylinder in a T-junction to drive fluid flow in microchannels. Paustian et al. 
(Paustian et al., 2014) used arrays of Janus micropillars to produce a pressure gradient in a microchannel under the AC electric field. Furthermore, Wu et al. (X. Wu, Ramiah Rajasekaran, \& Martin, 2016) fabricated a conical-pore polyethylene terephthalate conducting membrane immersed in an electrolyte and used it for micro pumping in microfluidics under AC electric field.

The ICEK micropumps introduced so far are categorized as ACEO micropumps where the fluid velocity in the order of 0.1-2.5 mm/s has been reported with applying DC electric signals (Lian \& Wu, 2009; Yang Ng et al., 2012). Huang et al. (C.-C. Huang et al., 2010) used ACEO micropumps functioning under AC voltage $(\mathrm{V}<1.5$ amplitude) to generate fluid velocity in microchannels as high as $1.3 \mathrm{~mm} / \mathrm{s}$. Among the existing ICEO micropumps, Nobari et al. (Nobari et al., 2016) demonstrated an average fluid velocity of $1750 \mu \mathrm{m} / \mathrm{s}$ using DC electric field with $\mathrm{E}=300 \mathrm{~V} / \mathrm{cm}$ strength in a microchannel. Altogether, both ACEO and ICEO micropumps can produce a wide range of fluid velocities and are exceptionally applicable for high-speed liquid pumping in microchannels.

\subsection{ICEK micromixers}

Rapid and homogeneous mixing of different solutions in microfluidics is vital for numerous LOC applications, such as biochemical reactions (S.-J. Kim, Wang, Burns, \& Kurabayashi, 2009), drug delivery (X. Jia et al., 2016), biological agent detection (Cho, Chung, Kim, Jung, \& Seo, 2015), and DNA hybridization (R. H. Liu, Lenigk, \& Grodzinski, 2003). Diffusion is the primary mechanism of fluid mixing in microscale due to the low convective mass transfer (M. K. D. Manshadi et al., 2019). Various types of passive and active micromixers have been developed to increase the contact surface area (interface) and reduce the mixing path, therefore enhancing the mixing in microchannels (Lee, Chang, Wang, \& Fu, 2011; Lee, Wang, Liu, \& $\mathrm{Fu}, 2016)$. Electrokinetic micromixers have demonstrated to be one of the most effective mixing methods in microfluidics where their performance is dependent on mixing time, length, and index (Lee et al., 2011; Rashidi, Bafekr, Valipour, \& Esfahani, 2018). ICEK electrokinetic-based micromixers, in specific, have shown their potential for active mixing in microchannels due to their high flexibility, controllability, and easy usage (Harnett, Templeton, Dunphy-Guzman, Senousy, \& Kanouff, 2008; Rashidi et al., 2018). The generated MVs around polarizable surfaces (electrodes or objects) increase chaotic movement in the electrolyte and therefore induce notable mixing. Similar to ICEK micropumps, two primary categories of ACEO and ICEO have shown high performance for microfluidic applications and are discussed below.

\subsubsection{ACEO micromixer}

The MVs produced around polarizable electrodes have been employed for the ACEO-based mixing of different solutions in microchannels. The first theoretical ACEO mixers were developed by Squires and Bazant (Bazant \& Squires, 2004; Todd M Squires \& Bazant, 2004) and Sasaki et al. (Sasaki, Kitamori, \& Kim, 2006), where they introduced a pair of meandering electrodes for rapid mixing of liquids inside microchannels. The results demonstrated $90 \%$ mixing efficiency, where one-millimeter length of mixing using the active ACEO was equivalent to $25 \mathrm{~mm}$ length of passive mixing using the diffusional mechanism. The effective mixing in this system lasts $0.18 \mathrm{~s}$ which was 20 folds faster than diffusional mixing. Kim et al. (B. J. Kim, Yoon, Sung, \& Smith, 2007) further employed asymmetric microelectrodes for simultaneous pumping and mixing within microfluidics, where the efficiency of $94.9 \%$ mixing was demonstrated under the flow rate of $0.27 \mathrm{~m} 2 / \mathrm{s}$. Yoon et al. (Yoon, Kim, \& Sung, 2008) employed asymmetric electrode arrays to generate pumping and mixing flow modes with $80 \%$ efficacy within $350 \mu \mathrm{m}$ mixing length under the flow rate of $0.01 \mathrm{~m} 2 / \mathrm{s}$. The performance of various ACEO micromixers developed so far is detailed inTable 1 .

\subsubsection{ICEO micromixer}

Following the suggestion of Squires and Bazant (Bazant \& Squires, 2004; Todd M Squires \& Bazant, 2004) on demonstrating the capability of MVs generated around polarizable objects for ICEO mixing, Harnett et al. (Harnett et al., 2008) proved this capability numerically and experimentally. A numerical model was developed to determine optimal design parameters of ICEO mixing followed by fabricating the optimal design array of gold-coated hurdles of the triangular cross-section in microchannels under a low-frequency $\mathrm{AC}$ field applied on the sidewalls. A rapid increase in mixing from zero to almost perfectly mixed state 
(100\%) in a small length scale was demonstrated (Fig. 3A ).

Wu and $\mathrm{Li}(\mathrm{Z} . \mathrm{Wu} \& \mathrm{Li}, 2008 \mathrm{~b})$ further simplified the design configuration to a triangle conducting hurdle pair on microchannel walls while enhanced the mixing performance. They optimized the system parameters and demonstrated $92 \%$ mixing efficiency. It was shown that the rectangular conducting objects placed on the microchannel walls generated the highest mixing efficiency (Fig. 3B ). Nevertheless, Jain et al. (Jain, Yeung, \& Nandakumar, 2009) showed that a pair of near tight triangle hurdle present even a better mixing performance as $99 \%$ efficiency. The performance of various ICEO micromixers developed so far are highlighted in Table 2 .

The ICEK Micromixers have become popular for LOC applications due to their mixing efficiency (above $90 \%$ ), simplicity and rapid mixing. However, the majority of studies are still limited to numerical studies to optimize electrodes shape and arrangement. Further experimental studies are needed to evaluate the performance of proposed mixing systems. Among various designs, the model of Daghighi and Li (Daghighi \& Li, 2013) (Fig. 3C ) is attractive due to its simple structure and straightforward fabrication method (Kazemi, Nourian, Nobari, \& Movahed, 2017; M. K. D. Manshadi et al., 2019; Shamloo, Madadelahi, \& Abdorahimzadeh, 2017). Further experimental characterization on this design configuration would guarantee an affordable and scalable technique for high-performance ICEK mixing within microfluidics.

\subsection{ICEK microvalves}

Microvalves are vital elements of complex microfluidic devices to control fluid flow along with a network of microchannels. Various types of microvalves have been developed for basic fluid flow mechanistic studies (Shoji \& Kawai, 2011), in vitro diagnostics (Tikka, Faulkner, \& Al-Sarawi, 2011), and biological sample delivery (Samiei, Tabrizian, \& Hoorfar, 2016). Rapid response and ability to be integrated into microfluidic systems are the essential criteria for selecting appropriate microvalves. The potential of ICEK phenomenon for controlling the direction of fluid flow through microchannels was first demonstrated by Sugioka (Sugioka, 2010). A rotating elliptical metal cylinder placed within a normally open microchannel was actuated using MVs around it under both DC and AC electric fields. This actuation induced the closure of the liquid flow in microchannels (Fig. 4A ). In another design, Daghighi and Li (Daghighi \& Li, 2011) utilized a Janus particle within a microchamber with three side channels, where the direction of fluid flow inside the channels was controlled under the motion of the Janus particle in the microchamber as a result of external electric field actuation (Fig. 4B ). Zhang et al. (Capretto, Cheng, Hill, \& Zhang, 2011) employed electrically a series of conducting surfaces embedded at the corners of a microchannel network to use ICEK actuation for controlling the flow direction toward desired outlets (Fig. 4C ). A similar technique was used by Wang et al. (C. Wang et al., 2016) to control fluid motion in a more complex fluidic network. Applying the electric field to the flow using conducting surfaces placed on a Y-junction wall generated MVs to control the flow direction toward the outlets (Fig. 4D ). Li et al. (Li \& Li, 2018) employed an electrically induced Janus droplet to produce ICEK microvalves functioning under DC electric field and to control fluid flow direction within a microchamber with several outlets (Fig. 4E ). The Janus droplet was made of an oil droplet coated with aluminum oxide nanoparticles. Among different ICEK microvalves, this new system was demonstrated to be highly controllable and easy to manipulate for adjusting the fluid flow in microfluidics.

Unlike many other On/Off or disposable valves used in microfluidic systems, most ICEK microvalves act

like a control valve to control the flow direction in microchannels. Among different electrode configurations used for ICEK microvalves, the polarizable plates patterned on the microchannel walls are shown to be a simple and effective ICEK method for controlling the fluid flow direction in microchannels (Figs. 4C and 4D ). The response time for switching the flow direction reaches down to one second with the capability of controlling the flow direction in desired branches (Capretto et al., 2011; Daghighi \& Li, 2011; Li \& Li, 2018). All ICEK microvalves so far functioned based on the ICEO mechanism. There have not been any ACEO-based microvalves yet proposed.

\subsection{ICEK for two-phase liquid flow systems}

Two-phase flow microfluidic devices have been widely used for chemical filtration, pharmacology and drug 
delivery (Azizian et al., 2019; Bazazi, Sanati-Nezhad, \& Hejazi, 2018). When a leaky dielectric droplet immersed in another immiscible leaky dielectric liquid, four symmetric MVs are induced inside and outside the droplet (Lin, Skjetne, \& Carlson, 2012). This phenomenon was first observed by Taylor (Taylor, 1966) in his experimental work. Small deformation of the droplet was predicted theoretically by calculating the exerted electrical stress on droplet-medium interface (Taylor, 1966). This deformation is generated due to the distribution of the interfacial polarization charge on the interface (Lin et al., 2012). The induced charge on the interface of the liquid and the generated MVs around the droplet have a resemblance to the generated MVs around polarizable solid cylinder immersed in the electrolyte under an external electric field (Bazant, 2015). Squires and Bazant (Todd M Squires \& Bazant, 2004) utilized this analogy to determine radial and azimuthal fluid velocities of the ICEO flow around a conducting cylinder. Jung et al. (Jung, Oh, \& Kang, 2008) calculated the amount of induced charge in a leaky dielectric water droplet oscillated between two fixed electrodes. Flittner and Přiby (Flittner \& Přibyl, 2017) proposed a mathematical model for such oscillatory behavior of water droplets. Wuzhang et al. (Wuzhang, Song, Sun, Pan, \& Li, 2015) investigated oil droplet motion in different ionic surfactant solutions and observed that an increase in surfactant concentration led to a higher droplet velocity due to the enhancement in surface charge density. It was also found that two MVs are generated around the oil droplet as a consequence of the redistribution of the mobile surface charges on the droplet surface (Daghighi, Gao, \& Li, 2011). Li et al. (Li \& Li, 2016a) studied this phenomenon more precisely and proposed an analytical presentation for the local zeta potential distribution on the oil droplet. Furthermore, they experimentally observed the accumulation of passivated aluminum nanoparticles on one side of the droplet, which confirmed the charge redistribution on the droplet ( $\mathrm{Li} \mathrm{\&} \mathrm{Li}, 2016 \mathrm{~b}$ ). Mori and Young (Mori \& Young, 2018) further improved the Taylor model (Taylor, 1966) and simulated the droplet deformation using electro-diffusion theory, as an essential tool for describing electrokinetic phenomena by considering the charge diffusion model.

Besides the above studies for describing ICEK occurrence in two-phase flows, this phenomenon has also been employed in various microfluidic applications, such as electric separation of droplets (Guo et al., 2010), droplet coalescence (Xiaodong Chen, Song, Li, \& Hu, 2015; Y. Jia et al., 2018), emulsion micro-pumping (Bhaumik, Roy, Chakraborty, \& DasGupta, 2014), controlling microdroplet generation (Azizian et al., 2019; Kamali \& Manshadi, 2016), regulating the micro reactions (Y. Jia et al., 2017), droplet separation (Li \& Li, 2017; K. Zhao \& Li, 2018), controlling multiphase flow systems (W. Liu et al., 2017), particle flow-focusing (Ren, Liu, Liu, et al., 2018), droplet motion in water-air and water-oil interfaces (C. Wang, Li, Song, Pan, \& Li, 2018; C. Wang, Song, Pan, \& Li, 2018a, 2018b), and microvalves (Li \& Li, 2018).

\subsection{Induced-charge electrophoresis (ICEP)}

The ICEP concept was first introduced by Bazant and Squires (Bazant \& Squires, 2004). It was suggested that asymmetries on polarizable objects such as polarizable Janus particles or tear shape objects immersed in an electrolyte result in non-uniform charge distribution on the surface. Therefore, ICEO on one part of the surface is dominant and drives the particle in the electrolyte under either AC or DC electric fields (Bazant \& Squires, 2004). Squires and Bazant (Todd M Squires \& Bazant, 2006) further studied the effective asymmetries on the conducting particle motion, including inhomogeneous surface properties on the particle, nearly and highly asymmetry objects, and symmetric body in an electric-field gradient.

The inhomogeneity in surface properties of particles was considered in some studies. Gangwal et al. (Gangwal, Cayre, Bazant, \& Velev, 2008) observed the motion of a surface-coated Janus particle perpendicular to the axis of the applied AC electric field. Kilic and Bazant (Kilic \& Bazant, 2011) then numerically validated such motions. Boymelgreen and Miloh (Boymelgreen \& Miloh, 2012) derived a theoretical presentation for the motion of inhomogeneous Janus particles. Daghighi et al. (Daghighi, Sinn, Kopelman, \& Li, 2013) experimentally characterized the motion of Janus particles under DC electric field and observed that particles, unlike under the AC field, are aligned with the axis of the DC electric field.

The particle motion under an electric field was further studied for different types of particle geometries, including non-spherical particles (Yariv, 2005), colloidal rods (Saintillan, Darve, \& Shaqfeh, 2006), striped micro rods (Rose, Meier, Dougherty, \& Santiago, 2007), cylindrical particles (H. Zhao \& Bau, 2007), and 
slender particles (Yariv, 2008). Characterizing the translational and rotational motions of these particles has shown that the rods or slender objects tend to align properly with the electric field (Saintillan, Darve, et al., 2006; Yariv, 2008).

The ICEP phenomenon was also studied within microfluidics, where the attraction and repulsion of particles from the channel walls were characterized in different conditions (Saintillan, 2008; Z. Wu \& Li, 2009). $\mathrm{Wu}$ et al. (Z. Wu, Gao, \& Li, 2009) numerically simulated the motion of an ideally polarizable particle in a microchannel and demonstrated that the velocity of these particles was equal to the electrophoretic velocity of a non-conducting particle. However, the polarizable particles were repelled from the microchannel wall by the generated MVs around them, transporting the particles toward the centerline. The ICEP motion of conducting particles has been used for developing microsensors (Gangwal et al., 2008) microvalves and microactuators, (Daghighi \& Li, 2011; Sugioka, 2010), micropumps (Nobari et al., 2016), micromixers (Daghighi \& Li, 2013), and stabilization of sedimenting rods (Saintillan, Shaqfeh, \& Darve, 2006). In most of these studies, spherical conducting particles were employed within microfluidics, though further investigation is needed to characterize how other particle shapes contribute to enhancing the performance of ICEK-based microfluidic systems.

\subsection{ICEK-based particle manipulation}

Particle manipulation is an urging demand in biology and analytical chemistry (Haghighi, Talebpour, \& Nezhad, 2018; Hassanpour-Tamrin et al., 2017; Khetani, Mohammadi, \& Nezhad, 2018). Among various active and passive methods used for particle manipulation within microfluidics, the micro-vortices induced around conducting surfaces have attracted the attention for particle/cell manipulation. Here, we categorize particle manipulation into particle/cell focusing, trapping, and sorting.

\subsubsection{Particle focusing}

Particle/cell focusing inside a microchannel is a crucial pre-step for downstream particle/cell counting, detection and sorting (Kung, Huang, Chong, \& Chiou, 2016; X. Wang, Zandi, Ho, Kaval, \& Papautsky, 2015). Jia et al. (Y. Jia, Ren, \& Jiang, 2015), for the first time, demonstrated particle focusing in generated MVs under the AC electric field around the planar floating electrode placed at the bottom of a microchannel (Fig. 5A ). Chen et al. (Xiaoming Chen et al., 2017) employed this focusing method for the pre-focusing of particles in different solutions prior to their dielectrophoretic separation. Song et al. (Song, Wang, Li, Pan, \& Li, 2016) demonstrated particle focusing under DC electric field at the desired location in the microchannel by the induced MVs around a polarizable plate located near the microchannel walls (Fig. 5B ). Ren et al. (Ren et al., 2016) developed a continuous-flow microfluidic device in which the desired number of focused particles were isolated at the target outlets using the generated MVs around asymmetric electrodes placed at the bottom of the microchannel (Fig. 5C ). Liu et al. (W. Liu et al., 2016) presented a flow-focusing device in which a suspension of particles was forcused within a cylindrical volume with a diameter range of 1-8 $\mu \mathrm{m}$ (nominal diameter of $4 \mu \mathrm{m}$ ) and conducted toward the desired outlet by the induced MVs around a floating electrode (Fig. 5D ).

\subsubsection{Particle trapping}

Particle trapping and enrichment have tremendous applications in clinical diagnostics (Hammarstrom, Nilson, Laurell, Nilsson, \& Ekstrom, 2014), water quality management (Zeng, Chen, Vedantam, Tzeng, \& Xuan, 2013) and drug development (Collins et al., 2015). The ICEK flow has also been utilized for particle/cell trapping. Yalcin et al. (Yalcin, Sharma, Qian, Joo, \& Baysal, 2011) were the first to demonstrate the capability in particle trapping under DC electric field using the ICEO MVs around the floating electrodes placed on the microchannel wall. The trapping of particles down to $500 \mathrm{~nm}$ in diameter was demonstrated under induced MVs. Ren et al. (Ren et al., 2015) developed a particle enrichment device in which the particles were trapped at the desired location within the microchannel by applying an electric field around a floating electrode (Fig. 6A ). Tao et al. (Tao et al., 2016) demonstrated that increasing in size of several floating electrodes or enlarging the width of a single floating electrode enhanced the trapping efficiency (Fig. 6B ). Harrison et al. (Harrison et al., 2015) utilized the two counter-rotating MVs generated around two 
dielectric corners of a reservoir-microchannel junction for concentrating the particles of $1 \mu \mathrm{m}$ diameter. Wu et al. (Y. Wu, Ren, Tao, Hou, \& Jiang, 2016) used a rotating electric field for trapping polystyrene (PS) microspheres with 5-20 $\mu \mathrm{m}$ in diameter on a central electrodes array (Fig. 6C ). Last but not the least, Zhao and Yang (Cunlu Zhao \& Yang, 2018) used ICEK-based micro-vortices around a polarizable plate located on the microchannel wall for the enrichment of 50-1900 nm fluorescent particles under combined AC/DC electric field (Fig. 6D).

\subsubsection{Particle sorting}

Particle sorting is an essential procedure for biological analysis and treatment (Gómez-Pastora, Karampelas, Bringas, Furlani, \& Ortiz, 2017), diagnostic (D. Kim, Luo, Arriaga, \& Ros, 2018), and targeted drug development (Antfolk, Kim, Koizumi, Fujii, \& Laurell, 2017). Although the ICEK flow demonstrated its high capability for particle/cell manipulation, there are a few researches with the focus on particle/cell sorting. Zhang and Li (F. Zhang \& Li, 2014) used a numerical model to simulate ICEO MVs around a pair of metal plates located on opposite sides of the microchannel walls and evaluated their ability to sort particles based on the applied electric field, polarizability and size of the particles. A similar model was developed to assess sorting of Janus particles (F. Zhang \& Li, 2015), where the model tuned dominant determinants of the particle's path through the microchannels (relative polarizability ratio and the size of Janus particles) to sort them by steering their streamline path to the desired branch outlets under DC electric field (F. Zhang $\& \mathrm{Li}, 2015)$. There are still limited numbers of studies on ICEK particle/cell manipulation, especially for particle trapping and sorting. Further experimental works and characterizations are needed to demonstrate the inexpensive, easy fabrication, and high-performance ICEK particle/cell manipulation.

\section{Conclusion}

This paper reviews the breakthrough in microfluidic-based applications of induced-charge electrokinetic (ICEK) phenomenon for developing various lab-on-chip (LOC) components, including micromixers, micropumps, and microvalves as well as applications in induced charge electrophoresis (ICEP) and particle/cell manipulation. The ICEK micropumps provided ultrafast pumping capability in a wide range of flow rates for LOC applications. The most efficient AC electroosmotic (ACEO) micropumps operate based on either biased AC/DC signals or three-dimensional stepped electrode arrays. The most efficient ICEO micropumps utilized a circular conducting cylinder for pumping purposes. The ACEO and ICEO micromixers made based on ICEK have shown above $90 \%$ mixing efficiency. The ACEO micromixers used mostly floating electrodes on the microchannel walls. Among various ICEK-based mixers, employing a conducting circular cylinder or a floating conducting particle inside a microchamber has been widely studied. However, further experimental characterization is needed to optimize its performance. Besides, ICEK microvalves can operate as both On/Off and control valves, which are highly desirable for microfluidic applications. Among the ICEK-based microvalves developed, the ones that employed conducting plates on the microchannel walls showed the fastest switching response. There is still no ACEO microvalve developed operating based on the ICEK.

Despite the broad scope of the applications for ICEK microfluidics, there are still challenges in technology scaling up. The primary challenge is the costly fabrication of $2 \mathrm{D}$ and $3 \mathrm{D}$ electrodes within microchannels. To address this challenge, new inexpensive, scalable, and easy to fabricate methods are to be developed to assemble electrodes with the microchannels. Extensive studies are still needed to enhance the efficiency of this method and reduce the power required to implement the system in a feasible point-of-care setting. For example, it would be worthy to characterize the effect of charged particles (conducting or Janus) on the ICEO flow and the generated MVs; the effect of conducting surface properties such as hydrophobic and hydrophilic surfaces on the slip velocity around the object; the role of ion concentrations in the electrolyte solution on ICEO flow; and the influence of polarizability of the induced charges on the object and the flow around it. Most studied have employed spherical particles in the ICEK microfluidic devices and the effect of particle shape has not been yet considered for investigating the ICEP systems. Also, further studies are needed to characterize the effect of different particle configurations on the performance of ICEP systems. Moreover, research works have to delve into the potential of ICEK for particle/cell manipulation. The results showed that the induced MVs in ACEO and ICEO flows could be utilized for focusing, trapping, and sorting 
of particles as small as $50 \mathrm{~nm}$ in size. However, the challenge of separating and sorting of defined particle size from a wide variety of particulates in complex biofluids needs further investigation.

\section{Acknowledgments}

We gratefully acknowledge the support of this work by the Natural Sciences and Engineering Research Council of Canada (NSERC).

\section{References}

Ajdari, A. (2000). Pumping liquids using asymmetric electrode arrays.Physical Review E, 61 (1), R45.

Alipanah, M., \& Ramiar, A. (2017). High efficiency micromixing technique using periodic induced charge electroosmotic flow: A numerical study. Colloids and Surfaces A: Physicochemical and Engineering Aspects, 524, 53-65.

Antfolk, M., Kim, S. H., Koizumi, S., Fujii, T., \& Laurell, T. (2017). Label-free single-cell separation and imaging of cancer cells using an integrated microfluidic system. Scientific reports, 7 , 46507.

Azarmanesh, M., Dejam, M., Azizian, P., Yesiloz, G., Mohamad, A. A., \& Sanati-Nezhad, A. (2019). Passive microinjection within high-throughput microfluidics for controlled actuation of droplets and cells.Scientific reports, 9 (1), 6723.

Azimi, S., Nazari, M., \& Daghighi, Y. (2017). Developing a fast and tunable micro-mixer using induced vortices around a conductive flexible link. Physics of Fluids, 29 (3), 032004.

Azizian, P., Azarmanesh, M., Dejam, M., Mohammadi, M., Shamsi, M., Sanati-Nezhad, A., \& Mohamad, A. A. (2019). Electrohydrodynamic formation of single and double emulsions for low interfacial tension multiphase systems within microfluidics. Chemical Engineering Science, 195 , 201-207. doi:10.1016/j.ces.2018.11.050

Barani, A., Paktinat, H., Janmaleki, M., Mohammadi, A., Mosaddegh, P., Fadaei-Tehrani, A., \& SanatiNezhad, A. (2016). Microfluidic integrated acoustic waving for manipulation of cells and molecules. Biosensors and Bioelectronics, 85 , 714-725.

Bazant, M. Z. (2011). Induced-charge electrokinetic phenomenaElectrokinetics and Electrohydrodynamics in Microsystems (pp. 221-297): Springer.

Bazant, M. Z. (2015). Electrokinetics meets electrohydrodynamics. Journal of Fluid Mechanics, 782 , 1-4.

Bazant, M. Z., Kilic, M. S., Storey, B. D., \& Ajdari, A. (2009). Towards an understanding of induced-charge electrokinetics at large applied voltages in concentrated solutions. Advances in colloid and interface science, 152 (1-2), 48-88.

Bazant, M. Z., \& Squires, T. M. (2004). Induced-charge electrokinetic phenomena: theory and microfluidic applications. Physical review letters, 92 (6), 066101.

Bazant, M. Z., \& Squires, T. M. (2010). Induced-charge electrokinetic phenomena. Current Opinion in Colloid \& Interface Science, 15 (3), 203-213.

Bazazi, P., Sanati-Nezhad, A., \& Hejazi, S. (2018). Wetting dynamics in two-liquid systems: Effect of the surrounding phase viscosity. Physical Review E, 97 (6), 063104.

Bhagat, A. A. S., Bow, H., Hou, H. W., Tan, S. J., Han, J., \& Lim, C. T. (2010). Microfluidics for cell separation. Medical \& biological engineering $\& 3$ computing, 48 (10), 999-1014.

Bhaumik, S. K., Roy, R., Chakraborty, S., \& DasGupta, S. (2014). Low-voltage electrohydrodynamic micropumping of emulsions. Sensors and Actuators B: Chemical, 193 , 288-293. doi:10.1016/j.snb.2013.11.082

Bown, M., \& Meinhart, C. (2006). AC electroosmotic flow in a DNA concentrator. Microfluidics and nanofluidics, 2 (6), 513-523. 
Boymelgreen, A. M., \& Miloh, T. (2012). Induced-charge electrophoresis of uncharged dielectric spherical J anus particles.Electrophoresis, 33 (5), 870-879.

Brown, A., Smith, C., \& Rennie, A. (2000). Pumping of water with ac electric fields applied to asymmetric pairs of microelectrodes. Physical Review E, 63 (1), 016305.

Capretto, L., Cheng, W., Hill, M., \& Zhang, X. (2011). Micromixing within microfluidic devices. Top Curr Chem, 304, 27-68. doi:10.1007/128_2011_150

Chen, J.-L., Shih, W.-H., \& Hsieh, W.-H. (2013). AC electro-osmotic micromixer using a face-to-face, asymmetric pair of planar electrodes.Sensors and Actuators B: Chemical, 188 , 11-21.

Chen, X., Ren, Y., Liu, W., Feng, X., Jia, Y., Tao, Y., \& Jiang, H. (2017). A simplified microfluidic device for particle separation with two consecutive steps: Induced charge electro-osmotic prefocusing and dielectrophoretic separation. Analytical chemistry, 89 (17), 9583-9592.

Chen, X., Song, Y., Li, D., \& Hu, G. (2015). Deformation and interaction of droplet pairs in a microchannel under ac electric fields. Physical Review Applied, 4 (2), 024005.

Cho, M., Chung, S., Kim, Y. T., Jung, J. H., \& Seo, T. S. (2015). A fully integrated microdevice for biobarcode assay based biological agent detection. Lab on a Chip, 15 (13), 2744-2748.

Collins, D. J., Morahan, B., Garcia-Bustos, J., Doerig, C., Plebanski, M., \& Neild, A. (2015). Twodimensional single-cell patterning with one cell per well driven by surface acoustic waves. Nature communications, 6,8686 .

Daghighi, Y., Gao, Y., \& Li, D. (2011). 3D numerical study of induced-charge electrokinetic motion of heterogeneous particle in a microchannel. Electrochimica Acta, 56 (11), 4254-4262. doi:10.1016/j.electacta.2011.01.083

Daghighi, Y., \& Li, D. (2011). Micro-valve using induced-charge electrokinetic motion of Janus particle. Lab on a Chip, 11 (17), 2929. doi:10.1039/c1lc20229d

Daghighi, Y., \& Li, D. (2013). Numerical study of a novel induced-charge electrokinetic micro-mixer. Analytica Chimica Acta, 763 , 28-37. doi:10.1016/j.aca.2012.12.010

Daghighi, Y., Sinn, I., Kopelman, R., \& Li, D. (2013). Experimental validation of inducedcharge electrokinetic motion of electrically conducting particles. Electrochimica Acta, 87, 270-276. doi:10.1016/j.electacta.2012.09.021

Debesset, S., Hayden, C., Dalton, C., Eijkel, J. C., \& Manz, A. (2004). An AC electroosmotic micropump for circular chromatographic applications. Lab on a Chip, 4 (4), 396-400.

Dehghan Manshadi, M. K., Khojasteh, D., Mohammadi, M., \& Kamali, R. (2016). Electroosmotic micropump for lab-on-a-chip biomedical applications. International Journal of Numerical Modelling: Electronic Networks, Devices and Fields, 29 (5), 845-858.

Du, K., Liu, W., Ren, Y., Jiang, T., Song, J., Wu, Q., \& Tao, Y. (2018). A High-Throughput Electrokinetic Micromixer via AC Field-Effect Nonlinear Electroosmosis Control in 3D Electrode Configurations.Micromachines, 9 (9), 432.

Flittner, R., \& Přibyl, M. (2017). Computational fluid dynamics model of rhythmic motion of charged droplets between parallel electrodes. Journal of Fluid Mechanics, 822 , 31-53.

Gangwal, S., Cayre, O. J., Bazant, M. Z., \& Velev, O. D. (2008). Induced-charge electrophoresis of metallodielectric particles.Physical review letters, 100 (5), 058302.

Gao, X., \& Li, Y. X. (2018). Ultra-fast AC electro-osmotic micropump with arrays of asymmetric ring electrode pairs in 3D cylindrical microchannel. Journal of Applied Physics, 123 (16), 164301. 
Gómez-Pastora, J., Karampelas, I., Bringas, E., Furlani, E. P., \& Ortiz, I. (2017). Computational Analysis of a Two-Phase Continuous-Flow Magnetophoretic Microsystem for Particle Separation from Biological Fluids Computer Aided Chemical Engineering (Vol. 40, pp. 1183-1188): Elsevier.

Guo, F., Ji, X.-H., Liu, K., He, R.-X., Zhao, L.-B., Guo, Z.-X., . . Z Zhao, X.-Z. (2010). Droplet electric separator microfluidic device for cell sorting. Applied Physics Letters, 96 (19), 193701.

Haghighi, F., Talebpour, Z., \& Nezhad, A. S. (2018). Towards fully integrated liquid chromatography on a chip: Evolution and evaluation. TrAC Trends in Analytical Chemistry, 105 , 302-337.

Hammarstrom, B. r., Nilson, B., Laurell, T., Nilsson, J., \& Ekstrom, S. (2014). Acoustic trapping for bacteria identification in positive blood cultures with MALDI-TOF MS. Analytical chemistry, 86 (21), 10560-10567.

Harnett, C. K., Templeton, J., Dunphy-Guzman, K. A., Senousy, Y. M., \& Kanouff, M. P. (2008). Model based design of a microfluidic mixer driven by induced charge electroosmosis. Lab on a Chip, 8 (4), 565-572.

Harrison, H., Lu, X., Patel, S., Thomas, C., Todd, A., Johnson, M., . . . Wang, J. (2015). Electrokinetic preconcentration of particles and cells in microfluidic reservoirs. Analyst, 140 (8), 2869-2875.

Hassanpour-Tamrin, S., Taheri, H., Mahdi Hasani-Sadrabadi, M., Hamed Shams Mousavi, S., Dashtimoghadam, E., Tondar, M., . . . Jacob, K. I. (2017). Nanoscale optoregulation of neural stem cell differentiation by intracellular alteration of redox balance. Advanced Functional Materials, 27 (38), 1701420.

Hilber, W., Weiss, B., Saeed, A., Holly, R., \& Jakoby, B. (2009). Density-dependent particle separation in microchannels using 3D AC-driven electro-osmotic pumps. Sensors and Actuators A: Physical, 156 (1), $115-120$.

Hu, Q., Guo, J., Cao, Z., \& Jiang, H. (2018). Asymmetrical Induced Charge Electroosmotic Flow on a Herringbone Floating Electrode and Its Application in a Micromixer. Micromachines, 9 (8), 391.

Hu, Q., Ren, Y., Liu, W., Chen, X., Tao, Y., \& Jiang, H. (2017). Fluid flow and mixing induced by ac continuous electrowetting of liquid metal droplet. Micromachines, 8 (4), 119.

Huang, C.-C., Bazant, M. Z., \& Thorsen, T. (2010). Ultrafast high-pressure AC electro-osmotic pumps for portable biomedical microfluidics. Lab on a Chip, 10 (1), 80-85.

Huang, K.-R., Hong, Z.-H., \& Chang, J.-S. (2014). Microfluidic mixing on application of traveling wave electroosmosis. European Journal of Mechanics-B/Fluids, 48 , 153-164.

Islam, N., \& Reyna, J. (2012). Bi-directional flow induced by an AC electroosmotic micropump with DC voltage bias. Electrophoresis, 33 (7), 1191-1197.

Jain, M., Yeung, A., \& Nandakumar, K. (2009). Induced charge electro osmotic mixer: Obstacle shape optimization. Biomicrofluidics, 3 (2), 022413.

Jain, M., Yeung, A., \& Nandakumar, K. (2010). Analysis of Electrokinetic Mixing Techniques Using Comparative Mixing Index. Micromachines, 1 (2), 36-47. doi:10.3390/mi1020036

Jain, M., Yeung, A., \& Nandakumar, K. (2010). Induced charge electro-osmotic concentration gradient generator.Biomicrofluidics, 4 (1), 014110.

Jia, X., Wang, W., Han, Q., Wang, Z., Jia, Y., \& Hu, Z. (2016). Micromixer based preparation of functionalized liposomes and targeting drug delivery. ACS medicinal chemistry letters, 7 (4), 429-434.

Jia, Y., Ren, Y., Hou, L., Liu, W., Deng, X., \& Jiang, H. (2017). Sequential Coalescence Enabled Two-Step Microreactions in Triple-Core Double-Emulsion Droplets Triggered by an Electric Field. Small, 13 (46), 1702188.

Jia, Y., Ren, Y., Hou, L., Liu, W., Jiang, T., Deng, X., . . . Jiang, H. (2018). Electrically controlled rapid release of actives encapsulated in double-emulsion droplets. Lab on a Chip, 18 (7), 1121-1129. 
Jia, Y., Ren, Y., \& Jiang, H. (2015). Continuous-flow focusing of microparticles using induced-charge electroosmosis in a microfluidic device with 3D AgPDMS electrodes. RSC Advances, 5 (82), 66602-66610. doi:10.1039/c5ra14854e

Jung, Y.-M., Oh, H.-C., \& Kang, I. S. (2008). Electrical charging of a conducting water droplet in a dielectric fluid on the electrode surface. Journal of colloid and interface science, 322 (2), 617-623.

Kamali, R., \& Manshadi, M. K. D. (2016). Numerical simulation of the leaky dielectric microdroplet generation in electric fields.International Journal of Modern Physics C, 27 (01), 1650012.

Kamali, R., Manshadi, M. K. D., \& Mansoorifar, A. (2016). Numerical analysis of non Newtonian fluid flow in a low voltage cascade electroosmotic micropump. Microsystem Technologies, 22 (12), 2901-2907.

Kazemi, S., Nourian, V., Nobari, M., \& Movahed, S. (2017). Two dimensional numerical study on mixing enhancement in micro-channel due to induced charge electrophoresis. Chemical Engineering and Processing: Process Intensification, 120 , 241-250.

Khetani, S., Mohammadi, M., \& Nezhad, A. S. (2018). Filter-based isolation, enrichment, and characterization of circulating tumor cells.Biotechnology and bioengineering, 115 (10), 2504-2529.

Kilic, M. S., \& Bazant, M. Z. (2011). Induced-charge electrophoresis near a wall. Electrophoresis, 32 (5), 614-628.

Kim, B. J., Yoon, S. Y., Sung, H. J., \& Smith, C. G. (2007). Simultaneous mixing and pumping using asymmetric microelectrodes. Journal of Applied Physics, 102 (7), 074513.

Kim, D., Luo, J., Arriaga, E. A., \& Ros, A. (2018). Deterministic Ratchet for Sub-micrometer (Bio) particle Separation. Analytical chemistry, 90 (7), 4370-4379.

Kim, S.-J., Wang, F., Burns, M. A., \& Kurabayashi, K. (2009). Temperature-programmed natural convection for micromixing and biochemical reaction in a single microfluidic chamber. Analytical chemistry, 81 (11), $4510-4516$.

Kim, S., Han, S.-I., Park, M.-J., Jeon, C.-W., Joo, Y.-D., Choi, I.-H., \& Han, K.-H. (2013). Circulating tumor cell microseparator based on lateral magnetophoresis and immunomagnetic nanobeads. Analytical chemistry, 85 (5), 2779-2786.

Kinahan, D. J., Mangwanya, F., Garvey, R., Chung, D. W. Y., Lipinski, A., Julius, L. A. N., . . . Ducrée, J. (2016). Automation of Silica Bead-based Nucleic Acid Extraction on a Centrifugal Lab-on-a-Disc Platform. Journal of Physics: Conference Series, 757 . doi:10.1088/1742-6596/757/1/012013

Kung, Y. C., Huang, K. W., Chong, W., \& Chiou, P. Y. (2016). Tunnel Dielectrophoresis for Tunable, Single-Stream Cell Focusing in Physiological Buffers in High-Speed Microfluidic Flows. Small, 12 (32), 4343-4348.

Kuo, C.-T., \& Liu, C.-H. (2008). A novel microfluidic driver via AC electrokinetics. Lab on a Chip, 8 (5), 725-733.

Lee, C.-Y., Chang, C.-L., Wang, Y.-N., \& Fu, L.-M. (2011). Microfluidic mixing: a review. International journal of molecular sciences, 12 (5), 3263-3287.

Lee, C.-Y., Wang, W.-T., Liu, C.-C., \& Fu, L.-M. (2016). Passive mixers in microfluidic systems: A review. Chemical Engineering Journal, 288, 146-160.

Lenshof, A., Magnusson, C., \& Laurell, T. (2012). Acoustofluidics 8: applications of acoustophoresis in continuous flow microsystems. Lab on a Chip, 12 (7), 1210-1223.

Li, M., \& Li, D. (2016a). Redistribution of mobile surface charges of an oil droplet in water in applied electric field. Advances in colloid and interface science, $236,142-151$. 
Li, M., \& Li, D. (2016b). Vortices around Janus droplets under externally applied electrical field. Microfluidics and nanofluidics, 20 (5), 79.

Li, M., \& Li, D. (2017). Separation of Janus droplets and oil droplets in microchannels by wall-induced dielectrophoresis. Journal of Chromatography A, 1501, 151-160.

Li, M., \& Li, D. (2018). Microvalve using electrokinetic motion of electrically induced Janus droplet. Analytica chimica acta, 1021, 85-94.

Lian, M., \& Wu, J. (2009). Ultrafast micropumping by biased alternating current electrokinetics. Applied Physics Letters, 94 (6), 064101.

Lin, Y., Skjetne, P., \& Carlson, A. (2012). A phase field model for multiphase electro-hydrodynamic flow. International Journal of Multiphase Flow, 45 , 1-11.

Liu, R. H., Lenigk, R., \& Grodzinski, P. A. (2003). Acoustic micromixer for enhancement of DNA biochip systems. Journal of Micro/Nanolithography, MEMS, and MOEMS, 2 (3), 178-185.

Liu, W., Ren, Y., Tao, Y., Chen, X., Yao, B., Hui, M., \& Bai, L. (2017). Control of two-phase flow in microfluidics using out-of-phase electroconvective streaming. Physics of Fluids, 29 (11), 112002.

Liu, W., Shao, J., Ren, Y., Liu, J., Tao, Y., Jiang, H., \& Ding, Y. (2016). On utilizing alternating current-flow field effect transistor for flexibly manipulating particles in microfluidics and nanofluidics.Biomicrofluidics, 10 (3), 034105.

Loucaides, N., Ramos, A., \& Georghiou, G. E. (2007). Novel systems for configurable AC electroosmotic pumping. Microfluidics and nanofluidics, 3 (6), 709-714.

Manshadi, M. K., Saadat, M., Mohammadi, M., Shamsi, M., Dejam, M., Kamali, R., \& Sanati-Nezhad, A. (2018). Delivery of magnetic micro/nanoparticles and magnetic-based drug/cargo into arterial flow for targeted therapy. Drug delivery, 25 (1), 1963-1973.

Manshadi, M. K. D., Khojasteh, D., Mansoorifar, A., \& Kamali, R. (2016). Efficiency enhancement of ICEK micromixer by a rectangular obstacle. Paper presented at the 3rd annual international conference on new research achievements in chemistry and chemical engineering. Ferdowsi University of Mashhad, Tehran Google Scholar.

Manshadi, M. K. D., Nikookar, H., Saadat, M., \& Kamali, R. (2019). Numerical analysis of non-uniform electric field effects on induced charge electrokinetics flow with application in micromixers. Journal of Micromechanics and Microengineering .

Mirzajani, H., Cheng, C., Wu, J., Ivanoff, C. S., Aghdam, E. N., \& Ghavifekr, H. B. (2016). Design and characterization of a passive, disposable wireless AC-electroosmotic lab-on-a-film for particle and fluid manipulation. Sensors and Actuators B: Chemical, $235,330-342$.

Mohammadi, M., Kinahan, D. J., \& Ducree, J. (2016). Lumped-Element Modeling for Rapid Design and Simulation of Digital Centrifugal Microfluidic Systems Lab-on-a-Chip Fabrication and Application .

Mohammadi, M., Madadi, H., Casals-Terre, J., \& Sellares, J. (2015). Hydrodynamic and direct-current insulator-based dielectrophoresis (H-DC-iDEP) microfluidic blood plasma separation. Analytical and bioanalytical chemistry, 407 (16), 4733-4744.

Morgan, H., Green, N. G., Ramos, A., \& Garcia-Sanchez, P. (2007). Control of two-phase flow in a microfluidic system using ac electric fields. Applied Physics Letters, 91 (25), 254107.

Mori, Y., \& Young, Y.-N. (2018). From electrodiffusion theory to the electrohydrodynamics of leaky dielectrics through the weak electrolyte limit. Journal of Fluid Mechanics, 855 , 67-130. 
Nobari, M., Movahed, S., Nourian, V., \& Kazemi, S. (2016). A numerical investigation of a novel micro-pump based on the induced charged electrokinetic phenomenon in the presence of a conducting circular obstacle. Journal of Electrostatics, 83 , 97-107.

Olesen, L. H., Bruus, H., \& Ajdari, A. (2006). ac electrokinetic micropumps: The effect of geometrical confinement, Faradaic current injection, and nonlinear surface capacitance. Physical Review E, 73 (5), 056313.

Park, B.-O., \& Song, S. (2012). Effects of multiple electrode pairs on the performance of a micromixer using dc-biased ac electro-osmosis. Journal of Micromechanics and Microengineering, 22 (11), 115034.

Paustian, J. S., Pascall, A. J., Wilson, N. M., \& Squires, T. M. (2014). Induced charge electroosmosis micropumps using arrays of Janus micropillars. Lab on a Chip, 14 (17), 3300-3312.

Pinon, M. V., Benitez, B. C., Pramanick, B., Perez-Gonzalez, V. H., Madou, M. J., Martinez-Chapa, S. O., \& Hwang, H. (2017). Direct current-induced breakdown to enhance reproducibility and performance of carbon-based interdigitated electrode arrays for AC electroosmotic micropumps. Sensors and Actuators A: Physical, 262 , 10-17.

Ramos, A., Garcia-Sanchez, P., \& Morgan, H. (2016). AC electrokinetics of conducting microparticles: A review. Current Opinion in Colloid \& Interface Science, 24, 79-90. doi:10.1016/j.cocis.2016.06.018

Ramos, A., Garcia, P., Gonzalez, A., Castellanos, A., Morgan, H., \& Green, N. G. (2005). AC electrokinetic pumping of liquids using arrays of microelectrodes. Paper presented at the Bioengineered and Bioinspired Systems II.

Ramos, A., Gonzalez, A., Castellanos, A., Green, N. G., \& Morgan, H. (2003). Pumping of liquids with ac voltages applied to asymmetric pairs of microelectrodes. Physical Review E, 67 (5), 056302.

Ramos, A., Morgan, H., Green, N. G., \& Castellanos, A. (1999). AC electric-field-induced fluid flow in microelectrodes. Journal of colloid and interface science, 217 (2).

Rashidi, S., Bafekr, H., Valipour, M. S., \& Esfahani, J. A. (2018). A review on the application, simulation, and experiment of the electrokinetic mixers. Chemical Engineering and Processing-Process Intensification, $126,108-122$.

Ren, Y., Liu, J., Liu, W., Lang, Q., Tao, Y., Hu, Q., . . . Jiang, H. (2016). Scaled particle focusing in a microfluidic device with asymmetric electrodes utilizing induced-charge electroosmosis. Lab on a Chip, 16 (15), 2803-2812.

Ren, Y., Liu, W., Jia, Y., Tao, Y., Shao, J., Ding, Y., \& Jiang, H. (2015). Induced-charge electroosmotic trapping of particles. Lab on a Chip, 15 (10), 2181-2191.

Ren, Y., Liu, W., Tao, Y., Hui, M., \& Wu, Q. (2018). On ac-field-induced nonlinear electroosmosis next to the sharp corner-field-singularity of leaky dielectric blocks and its application in on-chip micro-mixing. Micromachines, 9 (3), 102.

Ren, Y., Liu, X., Liu, W., Tao, Y., Jia, Y., Hou, L., . . . Jiang, H. (2018). Flexible particle flow-focusing in microchannel driven by droplet-directed induced-charge electroosmosis. Electrophoresis, 39 (4), 597-607.

Rose, K. A., Meier, J. A., Dougherty, G. M., \& Santiago, J. G. (2007). Rotational electrophoresis of striped metallic microrods. Physical Review E, 75 (1), 011503.

Rouabah, H. A., Park, B. Y., Zaouk, R. B., Morgan, H., Madou, M. J., \& Green, N. G. (2011). Design and fabrication of an ac-electro-osmosis micropump with 3D high-aspect-ratio electrodes using only SU-8. Journal of Micromechanics and Microengineering, 21 (3), 035018.

Sackmann, E. K., Fulton, A. L., \& Beebe, D. J. (2014). The present and future role of microfluidics in biomedical research. Nature, 507 (7491), 181-189. 
Saintillan, D. (2008). Nonlinear interactions in electrophoresis of ideally polarizable particles. Physics of Fluids, 20 (6), 067104.

Saintillan, D., Darve, E., \& Shaqfeh, E. S. (2006). Hydrodynamic interactions in the induced-charge electrophoresis of colloidal rod dispersions. Journal of Fluid Mechanics, 563 , 223-259.

Saintillan, D., Shaqfeh, E. S., \& Darve, E. (2006). Stabilization of a suspension of sedimenting rods by induced-charge electrophoresis.Physics of Fluids, 18 (12), 121701.

Samiei, E., Tabrizian, M., \& Hoorfar, M. (2016). A review of digital microfluidics as portable platforms for lab-on a-chip applications.Lab on a chip, 16 (13), 2376-2396.

Sasaki, N., Kitamori, T., \& Kim, H.-B. (2006). AC electroosmotic micromixer for chemical processing in a microchannel. Lab on a Chip, 6 (4), 550-554.

Sasaki, N., Kitamori, T., \& Kim, H.-B. (2010). Experimental and theoretical characterization of an AC electroosmotic micromixer.Analytical Sciences, 26 (7), 815-819.

Senousy, Y., \& Harnett, C. (2010). Fast three dimensional ac electro-osmotic pumps with nonphotolithographic electrode patterning.Biomicrofluidics, 4 (3), 036501.

Shamloo, A., Madadelahi, M., \& Abdorahimzadeh, S. (2017). Three-dimensional numerical simulation of a novel electroosmotic micromixer. Chemical Engineering and Processing: Process Intensification, 119 , 25-33. doi:10.1016/j.cep.2017.05.005

Shamsi, M., Mohammadi, A., Manshadi, M. K., \& Sanati-Nezhad, A. (2019). Mathematical and computational modeling of nano-engineered drug delivery systems. Journal of Controlled Release .

Shoji, S., \& Kawai, K. (2011). Flow control methods and devices in micrometer scale channels Microfluidics (pp. 1-25): Springer.

Song, Y., Wang, C., Li, M., Pan, X., \& Li, D. (2016). Focusing particles by induced charge electrokinetic flow in a microchannel.Electrophoresis, 37 (4), 666-675.

Squires, T. M. (2009). Induced-charge electrokinetics: fundamental challenges and opportunities. Lab on $a$ Chip, 9 (17), 2477. doi:10.1039/b906909g

Squires, T. M., \& Bazant, M. Z. (2004). Induced-charge electro-osmosis.Journal of Fluid Mechanics, 509 , $217-252$.

Squires, T. M., \& Bazant, M. Z. (2006). Breaking symmetries in induced-charge electro-osmosis and electrophoresis. Journal of Fluid Mechanics, 560 , 65-101.

Studer, V., Pepin, A., Chen, Y., \& Ajdari, A. (2002). Fabrication of microfluidic devices for AC electrokinetic fluid pumping. Microelectronic Engineering, 61, 915-920.

Studer, V., Pepin, A., Chen, Y., \& Ajdari, A. (2004). An integrated AC electrokinetic pump in a microfluidic loop for fast and tunable flow control. Analyst, 129 (10), 944-949.

Sugioka, H. (2010). High-speed rotary microvalves in water using hydrodynamic force due to induced-charge electrophoresis. Physical Review E, 81 (3), 036301.

Tang, M., Wang, G., Kong, S.-K., \& Ho, H.-P. (2016). A Review of Biomedical Centrifugal Microfluidic Platforms. Micromachines, 7 (2). doi:10.3390/mi7020026

Tao, Y., Ren, Y., Liu, W., Wu, Y., Jia, Y., Lang, Q., \& Jiang, H. (2016). Enhanced particle trapping performance of induced charge electroosmosis. Electrophoresis, 37 (10), 1326-1336.

Tatlısoz, M. M., \& Canpolat, Ç. (2018). Pulsatile flow micromixing coupled with ICEO for non-Newtonian fluids. Chemical Engineering and Processing-Process Intensification, 131 , 12-19. 
Tawfik, M. E., \& Diez, F. J. (2017). Maximizing fluid delivered by bubble-free electroosmotic pump with optimum pulse voltage waveform.Electrophoresis, 38 (5), 563-571.

Taylor, G. I. (1966). Studies in electrohydrodynamics. I. The circulation produced in a drop by an electric field. Proc. R. Soc. Lond. A, 291 (1425), 159-166.

Tikka, A. C., Faulkner, M., \& Al-Sarawi, S. F. (2011). Secure wireless actuation of an implanted microvalve for drug delivery applications.Smart Materials and Structures, 20 (10), 105011.

Urbanski, J. P., Thorsen, T., Levitan, J. A., \& Bazant, M. Z. (2006). Fast ac electro-osmotic micropumps with nonplanar electrodes.Applied Physics Letters, 89 (14), 143508.

Vigolo, D., Rusconi, R., Stone, H. A., \& Piazza, R. (2010). Thermophoresis: microfluidics characterization and separation. Soft matter, 6 (15), 3489-3493.

Wang, C., Li, M., Song, Y., Pan, X., \& Li, D. (2018). Electrokinetic motion of a spherical micro particle at an oil- water interface in microchannel. Electrophoresis, 39 (5-6), 807-815.

Wang, C., Song, Y., Pan, X., \& Li, D. (2016). A novel microfluidic valve controlledby induced charge electro-osmotic flow. Journal of Micromechanics and Microengineering, 26 (7), 075002. doi:10.1088/0960$1317 / 26 / 7 / 075002$

Wang, C., Song, Y., Pan, X., \& Li, D. (2018a). Electrokinetic motion of a submerged oil droplet near an air-water interface. Chemical Engineering Science, 192 , 264-272.

Wang, C., Song, Y., Pan, X., \& Li, D. (2018b). Electrokinetic Motion of an Oil Droplet Attached to a Water-Air Interface from Below. The Journal of Physical Chemistry B, 122 (5), 1738-1746.

Wang, X., Zandi, M., Ho, C.-C., Kaval, N., \& Papautsky, I. (2015). Single stream inertial focusing in a straight microchannel. Lab on a Chip, 15 (8), 1812-1821.

Wu, J. (2008). Ac electro-osmotic micropump by asymmetric electrode polarization. Journal of Applied Physics, 103 (2), 024907.

Wu, J., He, Z., Chen, Q., \& Lin, J.-M. (2016). Biochemical analysis on microfluidic chips. TrAC Trends in Analytical Chemistry, $80,213-231$.

Wu, X., Ramiah Rajasekaran, P., \& Martin, C. R. (2016). An alternating current electroosmotic pump based on conical nanopore membranes.ACS nano, 10 (4), 4637-4643.

Wu, Y., Ren, Y., Tao, Y., Hou, L., Hu, Q., \& Jiang, H. (2017). A novel micromixer based on the alternating current-flow field effect transistor. Lab on a Chip, 17 (1), 186-197.

Wu, Y., Ren, Y., Tao, Y., Hou, L., \& Jiang, H. (2016). Large-scale single particle and cell trapping based on rotating electric field induced-charge electroosmosis. Analytical chemistry, 88 (23), 11791-11798.

Wu, Z., Gao, Y., \& Li, D. (2009). Electrophoretic motion of ideally polarizable particles in a microchannel. Electrophoresis, 30 (5), 773-781.

Wu, Z., \& Li, D. (2008a). Micromixing using induced-charge electrokinetic flow. Electrochimica Acta, 53 (19), 5827-5835. doi:10.1016/j.electacta.2008.03.039

Wu, Z., \& Li, D. (2008b). Mixing and flow regulating by induced-charge electrokinetic flow in a microchannel with a pair of conducting triangle hurdles. Microfluidics and nanofluidics, 5 (1), 65-76.

Wu, Z., \& Li, D. (2009). Induced-charge electrophoretic motion of ideally polarizable particles. Electrochimica Acta, 54 (15), 3960-3967. doi:10.1016/j.electacta.2009.02.016

Wuzhang, J., Song, Y., Sun, R., Pan, X., \& Li, D. (2015). Electrophoretic mobility of oil droplets in electrolyte and surfactant solutions. Electrophoresis, 36 (19), 2489-2497. 
Yalcin, S. E., Sharma, A., Qian, S., Joo, S. W., \& Baysal, O. (2011). On-demand particle enrichment in a microfluidic channel by a locally controlled floating electrode. Sensors and Actuators B: Chemical, 153 (1), 277-283.

Yang, H., Jiang, H., Ramos, A., \& Garcia-Sanchez, P. (2009). AC electrokinetic pumping on symmetric electrode arrays. Microfluidics and nanofluidics, 7 (6), 767.

Yang Ng, W., Ramos, A., Cheong Lam, Y., \& Rodriguez, I. (2012). Numerical study of dc-biased acelectrokinetic flow over symmetrical electrodes. Biomicrofluidics, 6 (1), 012817.

Yariv, E. (2005). Induced-charge electrophoresis of nonspherical particles. Physics of Fluids, 17 (5), 051702.

Yariv, E. (2008). Slender-body approximations for electro-phoresis and electro-rotation of polarizable particles. Journal of Fluid Mechanics, 613 , 85-94.

Yoon, M. S., Kim, B. J., \& Sung, H. J. (2008). Pumping and mixing in a microchannel using $\mathrm{AC}$ asymmetric electrode arrays. International Journal of Heat and Fluid Flow, 29 (1), 269-280. doi:10.1016/j.ijheatfluidflow.2007.10.002

Yoshida, K., Sato, T., Eom, S. I., Kim, J.-w., \& Yokota, S. (2017). A study on an AC electroosmotic micropump using a square pole-Slit electrode array. Sensors and Actuators A: Physical, 265 , 152-160.

Zeng, J., Chen, C., Vedantam, P., Tzeng, T.-R., \& Xuan, X. (2013). Magnetic concentration of particles and cells in ferrofluid flow through a straight microchannel using attracting magnets. Microfluidics and nanofluidics, 15 (1), 49-55.

Zhang, F., \& Li, D. (2014). A novel particle separation method based on induced-charge electro-osmotic flow and polarizability of dielectric particles. Electrophoresis, 35 (20), 2922-2929.

Zhang, F., \& Li, D. (2015). Separation of dielectric Janus particles based on polarizability-dependent induced-charge electroosmotic flow. Journal of colloid and interface science, 448, 297-305.

Zhang, K., Mi, X., \& Sheng, B. (2013). Design of T-shaped micropump based on induced charge electroosmotic. Paper presented at the Abstract and Applied Analysis.

Zhang, K., Ren, Y., Hou, L., Feng, X., Chen, X., \& Jiang, H. (2018). An efficient micromixer actuated by induced-charge electroosmosis using asymmetrical floating electrodes. Microfluidics and nanofluidics, 22 (11), 130.

Zhang, K., Tian, F.-Z., \& Yu, M.-Z. (2012). Induced-charge electroosmosis around conducting and Janus cylinder in microchip. Thermal Science, 16 (5), 1502-1505.

Zhao, C. (2012). Induced-charge nonlinear electrokinetic phenomena and applications in micro/nano fluidics.

Zhao, C., \& Yang, C. (2012). Advances in electrokinetics and their applications in micro/nano fluidics. Microfluidics and nanofluidics, 13 (2), 179-203.

Zhao, C., \& Yang, C. (2013). Electrokinetics of non-Newtonian fluids: a review. Adv Colloid Interface Sci, 201-202 , 94-108. doi:10.1016/j.cis.2013.09.001

Zhao, C., \& Yang, C. (2018). Continuous-flow trapping and localized enrichment of micro-and nano-particles using induced-charge electrokinetics. Soft matter, 14 (6), 1056-1066.

Zhao, H., \& Bau, H. H. (2007). On the effect of induced electro-osmosis on a cylindrical particle next to a surface. Langmuir, 23 (7), 4053-4063.

Zhao, K., \& Li, D. (2018). Manipulation and separation of oil droplets by using asymmetric nano-orifice induced DC dielectrophoretic method.Journal of colloid and interface science, 512, 389-397.

Zhou, C., Zhang, H., Li, Z., \& Wang, W. (2016). Chemistry pumps: a review of chemically powered micropumps. Lab on a Chip, 16 (10), 1797-1811. 


\section{Table list:}

Table 1. The efficiency of AC electroosmotic (ACEO) micromixers demonstrated using either numerical $(\mathrm{N})$ or experimental (E) studies, or both.

\begin{tabular}{|c|c|c|c|c|c|}
\hline Authors & Research method & Mixing time (s) & $\begin{array}{l}\text { Mixing length } \\
(\mu \mathrm{m})\end{array}$ & Flow condition & Mixing index \\
\hline $\begin{array}{l}\text { Chen et al. } \\
\text { (J.-L. Chen, } \\
\text { Shih, \& Hsieh, } \\
\text { 2013) }\end{array}$ & $\mathrm{E}, \mathrm{N}$ & 0.75 & 64 & NA & $90 \%$ \\
\hline $\begin{array}{l}\text { Huang et al. } \\
\text { (K.-R. Huang, } \\
\text { Hong, \& } \\
\text { Chang, 2014) }\end{array}$ & $\mathrm{E}, \mathrm{N}$ & NA & 700 & $100(\mu \mathrm{m} / \mathrm{s})$ & $98 \%$ \\
\hline $\begin{array}{l}\text { Park and Song } \\
\text { (Park \& Song, } \\
2012 \text { ) }\end{array}$ & $\mathrm{E}$ & NA & 100 & $4(\mu \mathrm{L} / \min )$ & $95 \%$ \\
\hline $\begin{array}{l}\text { Hu et al. (Hu } \\
\text { et al., 2017) }\end{array}$ & $\mathrm{E}, \mathrm{N}$ & NA & 6,000 & $50(\mu \mathrm{L} / \min )$ & $85 \%$ \\
\hline $\begin{array}{l}\text { Wu et al. (Y. } \\
\text { Wu et al., } \\
2017 \text { ) }\end{array}$ & $\mathrm{E}, \mathrm{N}$ & NA & 2,300 & $2.16(\mu \mathrm{L} / \min )$ & $84 \%$ \\
\hline $\begin{array}{l}\text { Hu et al. (Hu, } \\
\text { Guo, Cao, \& } \\
\text { Jiang, 2018) }\end{array}$ & $\mathrm{N}$ & NA & 3,000 & NA & $98 \%$ \\
\hline $\begin{array}{l}\text { Zhang et al. } \\
\text { (Kailiang } \\
\text { Zhang et al., } \\
2018 \text { ) }\end{array}$ & $\mathrm{E}, \mathrm{N}$ & NA & 3,200 & $1,500(\mu \mathrm{m} / \mathrm{s})$ & $95 \%$ \\
\hline $\begin{array}{l}\text { Du et al. (Du } \\
\text { et al., 2018) }\end{array}$ & $\mathrm{N}$ & NA & NA & $10(\mathrm{~mm} / \mathrm{s})$ & $95 \%$ \\
\hline $\begin{array}{l}\text { Tatlisoz and } \\
\text { Canpolat } \\
\text { (Tatlısoz \& } \\
\text { Canpolat, } \\
\text { 2018) }\end{array}$ & $\mathrm{N}$ & 10 & 700 & NA & $96 \%$ \\
\hline
\end{tabular}

Table 2. Efficiency of different induced-charge electroosmosis (ICEO) micromixers for mixing liquids within microfluidics.

\begin{tabular}{llll}
\hline & $\begin{array}{l}\text { Object } \\
\text { type/position/Electric } \\
\text { field type }\end{array}$ & Mixing length $(\mu \mathrm{m})$ & Mixing efficiency (index) \\
\hline Authors & $\begin{array}{l}\text { Rectangular and right } \\
\text { triangle /near wall/DC }\end{array}$ & 2,000 & $\begin{array}{l}98 \% \text { mixing efficiency for } \\
\text { Anthony Yeung, \& } \\
\text { Krishnaswamy }\end{array}$ \\
\end{tabular}

Nandakumar, 2010) 


\begin{tabular}{|c|c|c|c|}
\hline Authors & $\begin{array}{l}\text { Object } \\
\text { type/position/Electric } \\
\text { field type }\end{array}$ & Mixing length $(\mu \mathrm{m})$ & Mixing efficiency (index) \\
\hline $\begin{array}{l}\text { Jain et al. (Mranal } \\
\text { Jain, Anthony Yeung, } \\
\text { \& K Nandakumar, } \\
\text { 2010) }\end{array}$ & Circular/near wall/DC & 1,000 & NA \\
\hline $\begin{array}{l}\text { Daghighi and Li } \\
\text { (Daghighi \& Li, 2013) }\end{array}$ & $\begin{array}{l}\text { Conducting } \\
\text { particle/free/DC }\end{array}$ & 80 & $100 \%$ \\
\hline $\begin{array}{l}\text { Manshadi et al. (M. K. } \\
\text { D. Manshadi et al., } \\
\text { 2016) }\end{array}$ & $\begin{array}{l}\text { Rectangular/near } \\
\text { wall/center-DC }\end{array}$ & 500 & $\begin{array}{l}95 \% \text { mixing efficiency } \\
\text { for near-wall object }\end{array}$ \\
\hline $\begin{array}{l}\text { Alipanahrostami and } \\
\text { Ramiar (Alipanah \& } \\
\text { Ramiar, 2017) }\end{array}$ & $\begin{array}{l}\text { Conducting edges /near } \\
\text { wall/AC }\end{array}$ & 20 & $99 \%$ \\
\hline $\begin{array}{l}\text { Azimi et al. (Azimi, } \\
\text { Nazari, \& Daghighi, } \\
\text { 2017) }\end{array}$ & $\begin{array}{l}\text { conductive flexible link } \\
\text { /near wall/DC }\end{array}$ & 156 & $90 \%$ \\
\hline $\begin{array}{l}\text { Kazemi et al. (Kazemi } \\
\text { et al., 2017) }\end{array}$ & $\begin{array}{l}\text { Conducting } \\
\text { particle/free/DC }\end{array}$ & 140 & $98 \%$ \\
\hline $\begin{array}{l}\text { Shamloo et al. } \\
\text { (Shamloo et al., 2017) }\end{array}$ & $\begin{array}{l}\text { Circular/triangular/square- } \\
\text { inside a } \\
\text { microchamber-DC }\end{array}$ & 2,000 & $\begin{array}{l}81 \% \text { mixing efficiency } \\
\text { for circular hurdle in a } \\
\text { rectangular chamber }\end{array}$ \\
\hline $\begin{array}{l}\text { Ren et al. (Ren, Liu, } \\
\text { Tao, Hui, \& Wu, 2018) }\end{array}$ & $\begin{array}{l}\text { semiconductor block } \\
\text { array/near wall/AC }\end{array}$ & 2,000 & $100 \%$ \\
\hline $\begin{array}{l}\text { Manshadi et al. (M. K. } \\
\text { D. Manshadi et al., } \\
\text { 2019) }\end{array}$ & $\begin{array}{l}\text { Circular/inside a } \\
\text { microchamber/DC }\end{array}$ & 500 & $96.89 \%$ \\
\hline
\end{tabular}

\section{Figure list:}

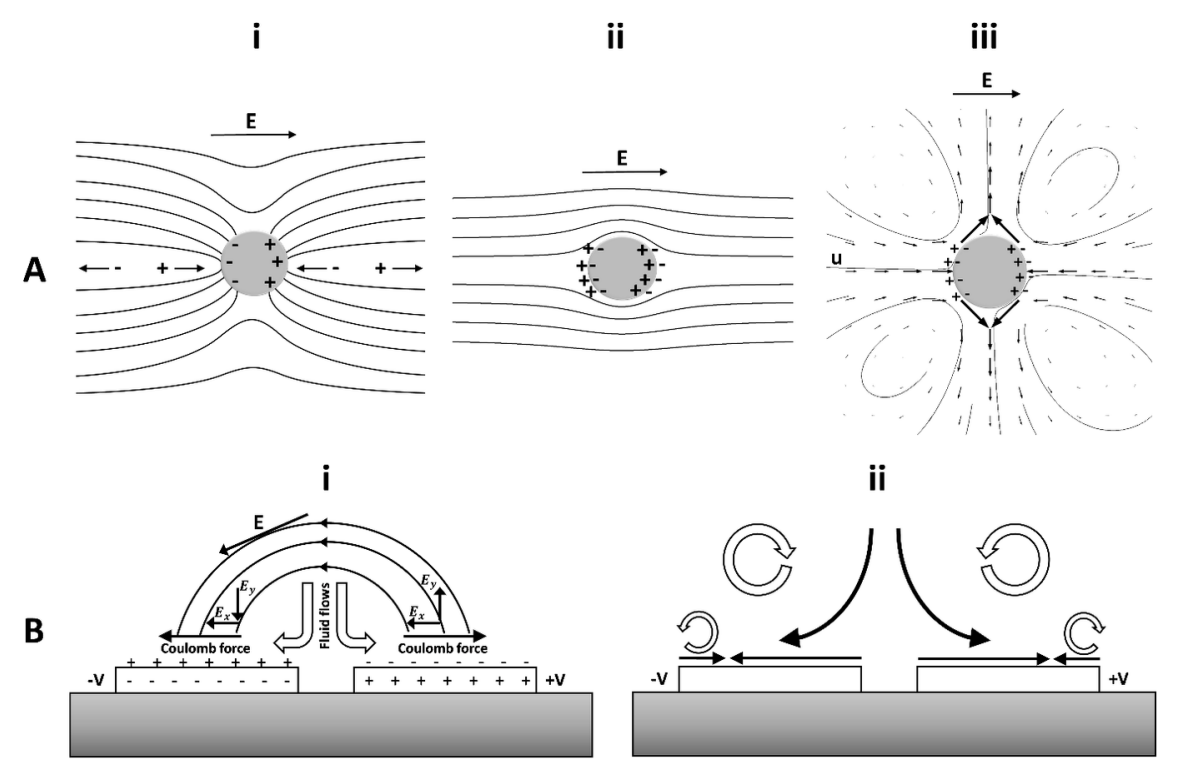


Figure 1. Non-linear Induced-charge electrokinetic (ICEK) phenomena. A) Polarizable cylinder, i) applying an electric field, ii) induced potential in the object, andiii) induced micro vortices around the object. B)Polarizable electrode: i) the induced electric potential,ii) the generated micro vortices around the electrode.

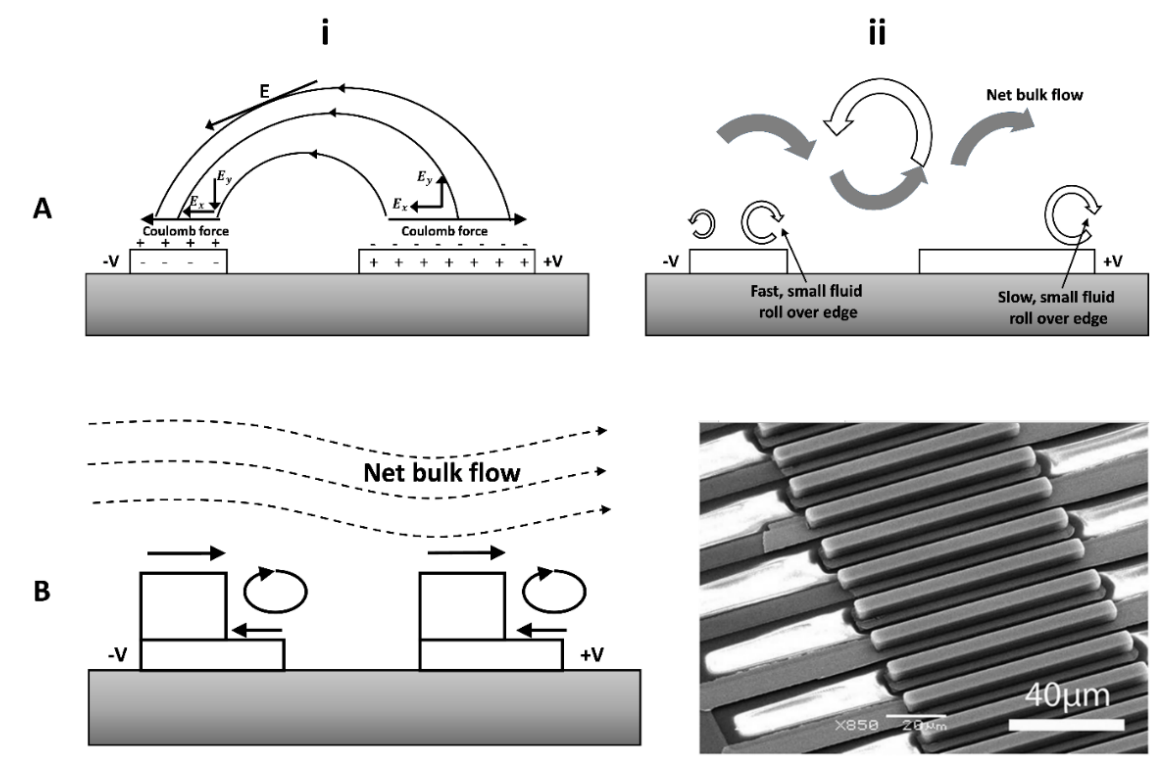

Figure 2 . Schematic view of AC electroosmotic (ACEO) micropumps to induce fluid flow in microchannel by (A ) a planar array of electrodes with different edge sizes s (Brown et al., 2000; V Studer et al., 2002) and (B ) a nonplanar configuration of 3D stepped electrodes (Urbanski et al., 2006). 

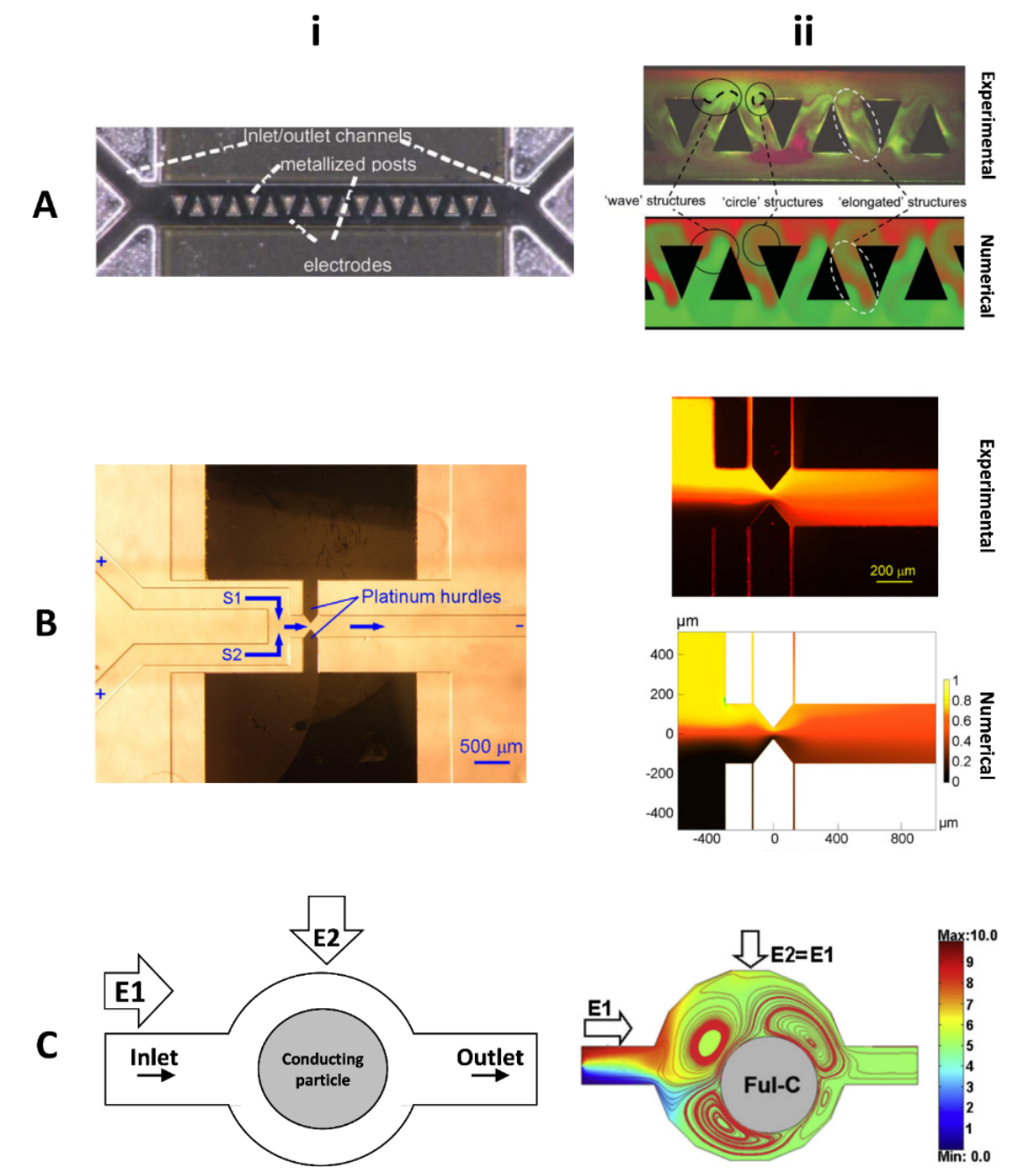

Figure 3. The schematics of induced-charge electroosmosis (ICEO) micromixers, (A) numerical and experimental results for rapid mixing state from zero to $100 \%$ under AC electric field applied on the sidewalls, (B) symmetry pair of triangle conducting hurdles on microchannel walls for inducing micromixing (Z. Wu \& Li, 2008a), (C) fully conducting particles in the micro-chamber for inducing micromixing (Daghighi \& Li, 2013). 

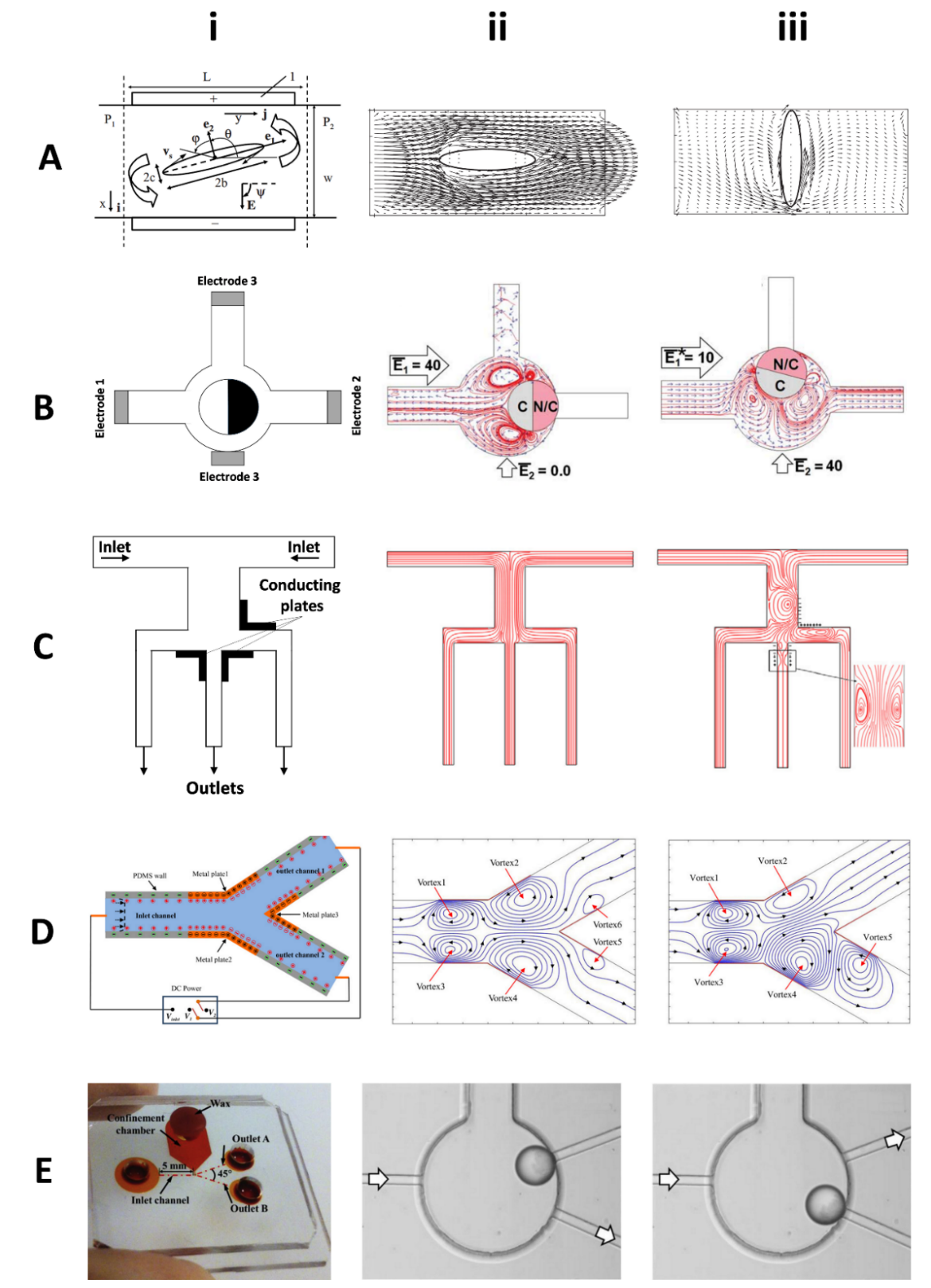

Figure 4. The ICEK microvalves utilizing, (A) rotary elliptical metal cylinders as an actuator within a microchannel (Sugioka, 2010), (B) a Janus particle actuated within a microchamber with three-side channels (Daghighi \& Li, 2011),(C) electrically conducting surfaces placed at the corners of a microchannel network for generating actuation MVs, (D)Y-junction walls for generating actuation MVs (C. Wang et al., 2016), and (E) electrically induced Janus droplet actuated within a chamber with one inlet and two outlets (Li \& $\mathrm{Li}, 2018)$. 


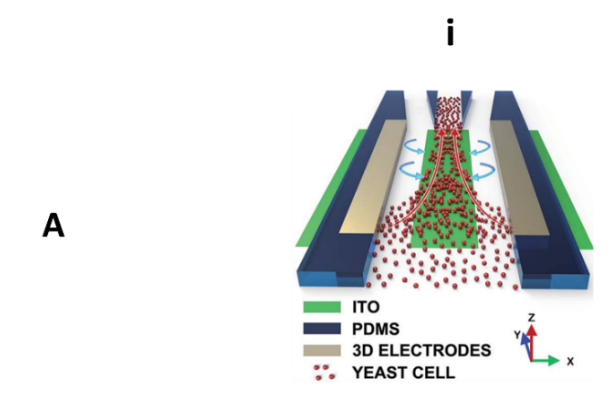

ii

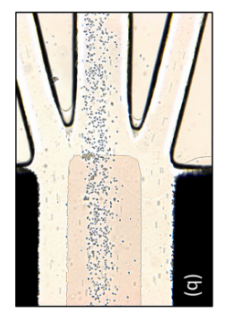

B
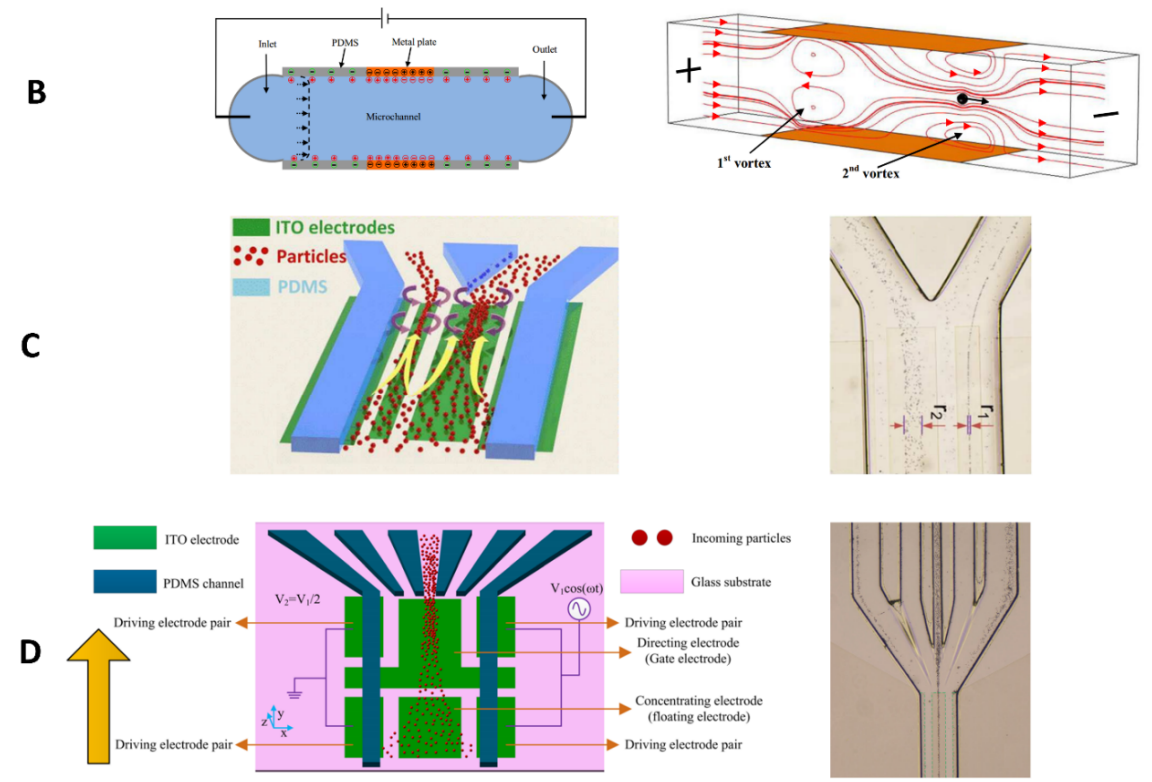

Figure 5. Different electrode design configurations in ICEK to generate MVs for particle focusing. (A) planar floating electrode placed at the bottom of the microchannel (Xiaoming Chen et al., 2017), (B) polarizable plates placed near the microchannel walls (Song et al., 2016), (C) asymmetric electrodes placed at the bottom of the microchannel (Ren et al., 2016), (D ) a floating electrode placed in the flow-focusing device (W. Liu et al., 2016). 

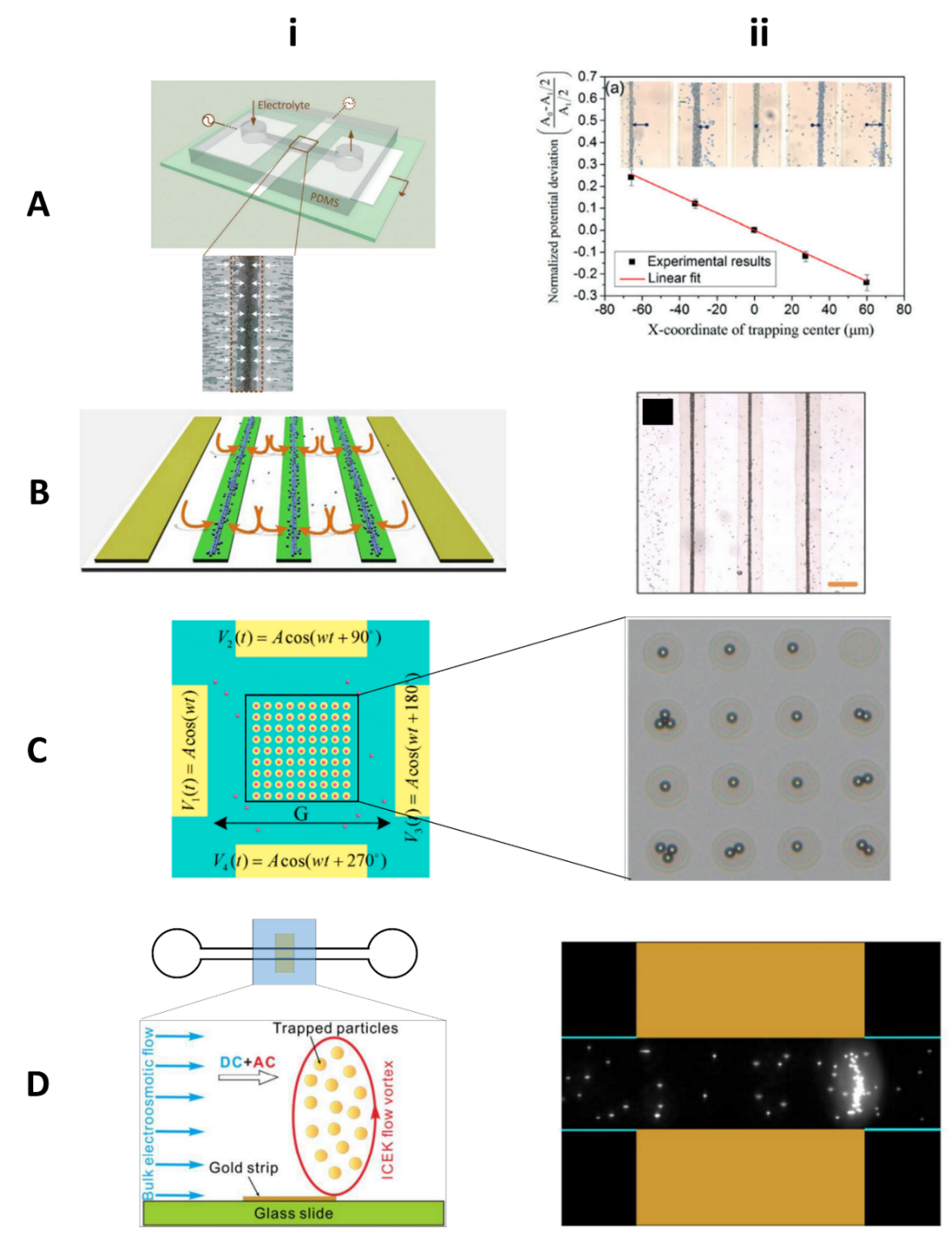

Figure 6. Different electrode design configurations in ICEK to generate MVs for particle trapping. (A) a floating electrode array for trapping $500 \mathrm{~nm}$ particles (Ren et al., 2015), (B)multiple floating electrodes (Tao et al., 2016), (C) rotating electric field and central electrodes for trapping 5-20 $\mu \mathrm{m}$ polystyrene (PS) particles (Y. Wu et al., 2016), (D) polarizable plate located on the microchannel wall for enrichment of 50-1900 nm particles (Cunlu Zhao \& Yang, 2018). 


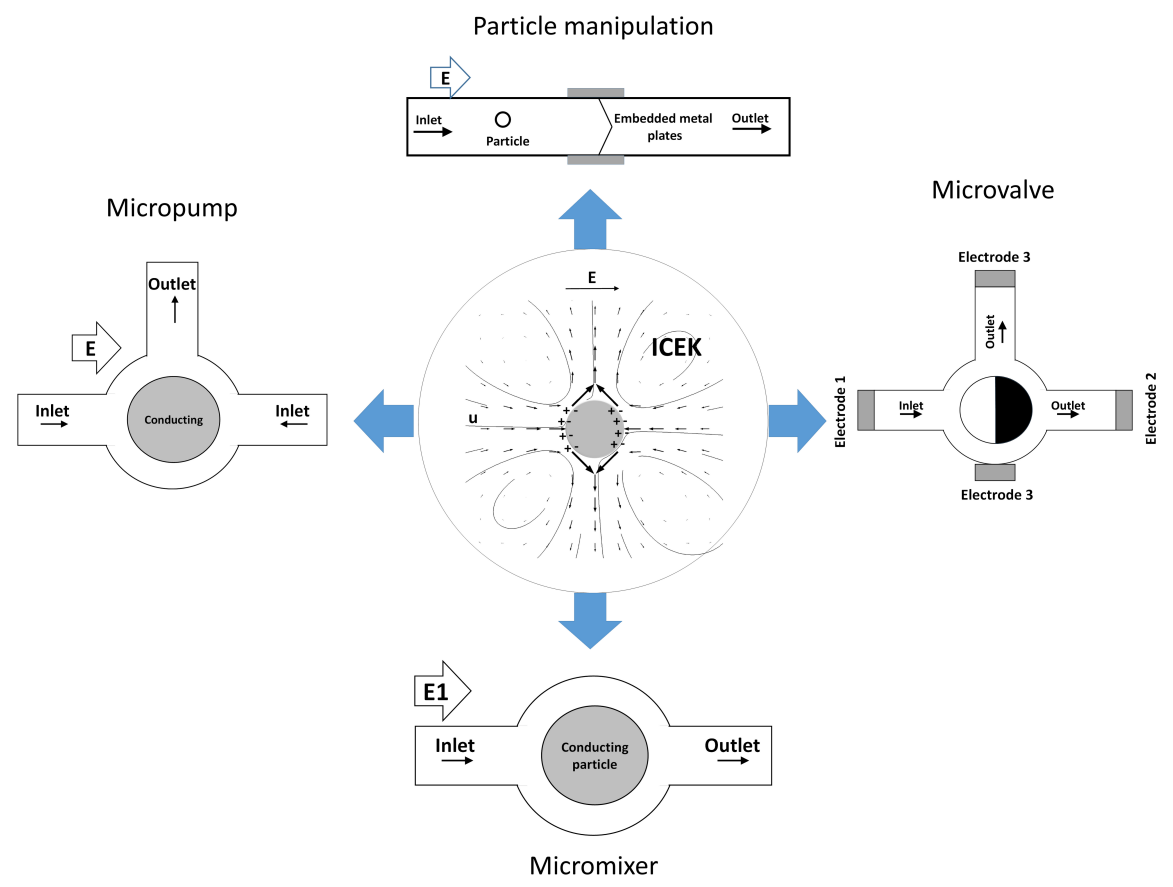

\section{Hosted file}

Figure 1 600dpi.TIF available at https://authorea.com/users/286547/articles/410830-inducedcharge-electrokinetic-icek-development-and-applications-in-microfluidics

\section{Hosted file}

Figure 2 600dpi.TIF available at https://authorea.com/users/286547/articles/410830-inducedcharge-electrokinetic-icek-development-and-applications-in-microfluidics

\section{Hosted file}

Figure 3 300dpi.TIF available at https://authorea.com/users/286547/articles/410830-inducedcharge-electrokinetic-icek-development-and-applications-in-microfluidics

\section{Hosted file}

Figure 4 300dpi.TIF available at https://authorea.com/users/286547/articles/410830-inducedcharge-electrokinetic-icek-development-and-applications-in-microfluidics

\section{Hosted file}

Figure 5 300dpi.TIF available at https://authorea.com/users/286547/articles/410830-inducedcharge-electrokinetic-icek-development-and-applications-in-microfluidics

\section{Hosted file}

Figure 6 300dpi.TIF available at https://authorea.com/users/286547/articles/410830-inducedcharge-electrokinetic-icek-development-and-applications-in-microfluidics 